\title{
3D Genome Analysis Identifies Enhancer Hijacking Mechanism for High-Risk Factors in Human T-Lineage Acute Lymphoblastic Leukemia
}

Lu Yang ${ }^{1,2,3}$, Fengling Chen ${ }^{4} \wedge$, Haichuan Zhu ${ }^{1,2,3 \wedge}$, Yang Chen ${ }^{4}$, Bingjie Dong ${ }^{1,2,3}$, Minglei Shi ${ }^{4}$, Weitao Wang $^{1,2,3}$, Qian Jiang ${ }^{6}$, Leping Zhang ${ }^{7}$, Xiaojun Huang ${ }^{2,6 *}$, Michael Q. Zhang ${ }^{4,5,8_{*}}$ and Hong $\mathrm{Wu}^{1,2,3,6 *}$

${ }^{1}$ The MOE Key Laboratory of Cell Proliferation and Differentiation, School of Life Sciences, ${ }^{2}$ Peking-Tsinghua Center for Life Sciences and ${ }^{3}$ Beijing Advanced Innovation Center for Genomics, Peking University, Beijing 100871, China; ${ }^{4}$ MOE Key Laboratory of Bioinformatics, Center for Synthetic and Systems Biology, Bioinformatics Division, BNRist, Department of Automation, ${ }^{5}$ School of Medicine, Tsinghua University, Beijing 100084, China; ${ }^{6}$ Peking University Institute of Hematology, National Clinical Research Center for Hematologic Disease, ${ }^{7}$ Department of Pediatric Hematology/Oncology, Peking University People's Hospital, Beijing 100044, China; ${ }^{8}$ Department of Biological Sciences, Center for Systems Biology, The University of Texas, Dallas 800 West Campbell Road, RL11, Richardson, TX 75080-3021, USA

$\wedge$ These authors contributed equally to this work.

${ }^{\S}$ Current address: Institute of Biology and Medicine, College of Life and Health Sciences, Wuhan University of Science and Technology, Hubei 430081, China.

${ }^{*}$ Corresponding authors:

hongwu@pku.edu.cn; xjhrm@medmail.com.cn; michael.zhang@utdallus.edu

Lead Contact: hongwu@pku.edu.cn 
Recent studies have demonstrated that 3D genome alterations play important roles in tumorigenesis ${ }^{1-3}$, including the development of hematological malignancies ${ }^{4-7}$. However, how such alterations may provide key insights into T-lineage acute lymphoblastic leukemia (T-ALL) patients is largely unknown. Here, we report integrated analyses of 3D genome alterations and differentially expressed genes (DEGs) in 18 newly diagnosed T-ALL patients and 4 healthy T cell controls. We found that 3D genome organization at the compartment, topologically associated domains (TAD) and loop levels as well as the gene expression profiles could hierarchically classify different subtypes of T-ALL according to the $\mathrm{T}$ cell differentiation trajectory. Alterations in the 3D genome were associated with nearly $45 \%$ of the upregulated genes in T-ALL. We also identified 34 previously unrecognized translocations in the noncoding regions of the genome and 44 new loops formed between translocated chromosomes, including translocation-mediated enhancer hijacking of the HOXA cluster. Our analysis demonstrated that T-ALLs with HOXA cluster overexpression were heterogeneous clinical entities, and ectopic expressions of the HOXA11-A13 genes, but not other genes in the HOXA cluster, were associated with immature phenotypes and poor outcomes. Our findings highlight the potentially important roles of 3D genome alterations in the etiology and prognosis of T-ALL.

Keywords: 3D genome alterations in T-ALL, chromosomal translocation, enhancer hijacking, ectopic HOXA11-A13 expression, poor prognosis of T-ALL. 
T-ALL is an aggressive hematological malignancy caused by genetic and epigenetic alterations that affect normal $\mathrm{T}$ cell development ${ }^{8}$. Recent whole-exome and RNA sequencing analyses of large T-ALL cohorts are focused on the coding region of the genome and have identified novel driver mutations, dysregulated transcription factors and pathways in T-ALLs ${ }^{9-12}$. To determine whether alterations in the $3 \mathrm{D}$ genome organization are associated with malignant transformation of T-ALL, we conducted $\mathrm{BL}-\mathrm{Hi}-\mathrm{C}^{13}$ analysis using purified primary leukemic blasts from 18 newly diagnosed T-ALL patients, including 8 early T-cell precursor ALL (ETP ALL) and 10 non-ETP ALL, two clinical subtypes of T-ALL, as well as normal T cells from 4 healthy volunteers (Supplementary Fig. 1a). The maximum resolutions of the chromatin contact maps for ETP, non-ETP ALL and normal samples were approximately 3.5, 3.5 and $10 \mathrm{~kb}$, respectively (Supplementary Table 1 ).

Principal component analysis (PCA) at the levels of the compartment, TAD and loop structures demonstrated that the T-ALL samples could be separated from the control samples by PC1, while ETP and non-ETP ALL could be separated by PC2 at all three architectural levels (Fig. 1a, upper panels) and be further delineated by hierarchical clustering analysis (Fig. 1a, lower panels). Detailed comparisons of the 3D chromosomal organizations of the T-ALL patients and the healthy controls revealed $1.59 \%$ of A-to-B and $1.38 \%$ of B-to-A T-ALL-associated switches at the compartment level (Supplementary Fig. 1b), 11\% ( 377/3421 ) T-ALL-specific TAD boundaries (Supplementary Fig. 1c), and 6\% (2330/38464) enhanced and 11\% (4073/38464) reduced loops (Supplementary Fig. 1d), implying that the global 3D 
genome alterations could link to the etiology of T-ALL development.

RNA-seq analysis was also performed on each sample to investigate the impact of 3D genome alterations on gene expression in the T-ALLs. PCA and hierarchical clustering showed that the transcriptome changes were highly consistent with the corresponding 3D genome structural changes (comparing Fig. 1a and $1 \mathrm{~b}$ ) and hence could serve as a biological readout for the functional impact of 3D genome alterations in T-ALL development.

Approximately 29\% (996/3392) of the DEGs were associated with 3D genome alterations (Supplementary Table 2). Among them, the genes associated with the B-to-A compartment switches, increased domain scores (D-score) and the corresponding enhanced loops were mostly upregulated (Fig. 1c, red bars and Fig. 1d), and were functionally enriched in pathways such as hematopoietic cell lineage, transcriptional misregulation in cancer and cell cycle (Fig. 1e). In contrast, the genes associated with the A-to-B compartment switches, decreased D-scores and reduced loops were mostly downregulated (Fig. 1c, blue bars and Fig. 1d), and these genes were enriched in pathways such as cytokine-cytokine receptor interaction and $\mathrm{T}$ cell receptor signaling (Fig. 1e).

Among the upregulated DEGs, CDK6 is a potential target for T-ALL treatment ${ }^{14}$. The CDK6 locus exhibited a strong intra-TAD interaction, and its expression was upregulated in all T-ALL samples (Fig. 1f; Supplementary Fig. 1e). Similarly, several upregulated oncogenic driver genes and T-ALL-associated transcription factors, such as MYB, MYCN, BCL11A, SOX4 and WT1, also had increased D-scores 
(Supplementary Fig. 1f).

While the majority of the DEGs were associated with structural changes at 1 or 2 levels, transcription factor SOX4,WT1 and TFDP2 had structural alterations at all 3 levels (Fig. 1g, Supplementary Fig. 1g and Supplementary Table 2). Importantly, when RcisTarget ${ }^{15}$ was used to predict the key transcription factors that might control the upregulated genes in the T-ALLS, SOX4,WT1 and TFDP2 were among the top-ranking candidates that potentially regulated approximately $28 \%$ of the upregulated genes (Supplementary Table 3). After eliminating the overlapping upregulated DEGs, we estimated that 3D genome alterations could associate with approximately $45 \%$ of the upregulated genes in T-ALL, of which $56 \%$ could be directly related to structural alterations, while the rest could be due to structure alteration-mediated dysregulated transcription factors.

ETP ALL is a subclass of T-ALL stalled at the early T progenitor stage, while non-ETP ALLs are blocked at the later T cell differentiation stages ${ }^{16}$. Our 3D genome landscape analyses could separate the ETP ALL samples from the non-ETP ALL samples (Fig. 1a), suggesting that the chromosomal organizations of T-ALL may represent different "frozen stages" of T cell development ${ }^{17}$. To test this hypothesis, we projected the T-ALL samples onto the $\mathrm{T}$ cell developmental trajectory defined by RNA-seq analysis ${ }^{18}$. PCA revealed that most of the ETP ALL samples were arrested at the immature stage, corresponding to the LMPP to Thy1 stages, while the non-ETP samples were arrested at the Thy2 to Thy4 stages (Fig. 2a). Since the ETP and non-ETP ALLs can be better separated at the loop level (Fig. 1a), we further analyzed 
114 the differences in loop structures between ETP and non-ETP ALL samples and

115 identified 1820 enhanced and 831 reduced loops in ETP (Fig. 2b). When plotting gene

116 expression changes between ETP and non-ETP ALL against the combined p-value of

117 the loop strength and D-score changes, we found a strong positive correlation

118 (Pearson's correlation coefficient 0.685; Fig. 2c). Approximately $20 \%$ and $16 \%$ of the

119 upregulated genes in ETP and non-ETP ALL, respectively, harbored chromatin

120 structure changes, including key transcription factors or oncogenes, such as CEBPA,

121 MYCN and LYL1 for ETP and LEF1, TCF12 and PAX9 for non-ETP ALL (Fig. 2c and

122 Supplementary Table 4).

123 Gene ontology analysis further revealed that genes associated with the ETP ALL

124 enhanced loops were enriched in immune response-activating signal transduction,

125 myeloid cell differentiation and regulation of $B$ cell activation, consistent with the

126 definition of ETP ALL (Fig. 2d). Genes associated with the non-ETP ALL enhanced

127 loops were enriched in terms such as positive regulation of RNA metabolism,

128 transcription, and TCR $\mathrm{V}(\mathrm{D}) \mathrm{J}$ recombination (Fig. $2 \mathrm{~d}$ ). The lack of TCR rearrangement

129 in most of the ETP ALL samples (Supplementary Fig. 2a) and different

130 rearrangements in individual non-ETP samples (Supplementary Fig. 2b) could be

131 easily observed from the Hi-C maps (Fig. 2e). Sample 093 was a unique case as it fell

132 between ETP and non-ETP ALL (Fig. 1a and 1b; Fig. 2a) and had significant TCR

133 rearrangement (Supplementary Fig. 2a). We also observed a lack of RAG1 and

134 PTCRA expression in most of the ETP ALL samples, which are essential for TCR

$135 V(D) J$ rearrangements (Fig. 2f). 
The 3D genome alteration analysis also provided a potential explanation for ETP and non-ETP ALL-specific transcription factor expressions. For example, we detected subtype-specific loops and expression patterns in the MEF2C locus in ETP and the

PAX9 locus in the non-ETP ALL samples, respectively, which were associated with

$1412 \mathrm{~g}$ and $2 \mathrm{~h})$.

Chromosomal translocation is one of the major driving forces for tumorigenesis ${ }^{19}$, especially for leukemia ${ }^{8}$. By adapting hic_breakfinder ${ }^{20}$, we identified 46

144 translocations in 14/18 T-ALL samples (Supplementary Table 5), of which 34 were novel events and 26 were interchromosomal events (Fig. 3a, red lines). Among 78 unique breakpoints identified, $47 \%$ were located in noncoding regions, and $66 \%$ were

147 located in the stable A compartment (Supplementary Fig. 3a). These newly identified

148 translocations not only influenced the expression of the nearest genes (Fig. 3a) but

149 also resulted in the formation of 44 new loops across the translocated chromosomes, 150 which we named translocation-mediated loops (Supplementary Fig. 3b; 151 Supplementary Table 6). Interestingly, the ends of these translocation-mediated loops 152 tend to anchor at the pre-existing loop anchors and CTCF binding sites (Fig. 3b). 153 Importantly, nearly $78 \%$ of the translocation-mediated loops with CTCF motifs were 154 linked to pairs of convergently orientated CTCF motifs (Fig. 3c), indicating that these 155 loops may be mediated by loop extrusion mechanism ${ }^{21-23}$.

156 We then investigated the potential mechanisms underlying 157 translocation-mediated T-ALL classification and gene activation. Clinically, non-ETP 
158 ALL can be further classified into the HOXA, TLX and TAL subtypes according to their

159 gene expression profiles ${ }^{24}$. Notably, there was a complete match between loop-based

160 hierarchical clustering and T-ALL subtypes, which were signified by chromosomal

161 translocation-mediated dysregulation of T-ALL-associated transcription factors (Fig.

$1623 d)$.

163 Translocations can activate T-ALL-associated transcription factors via either "cis"

164 or "trans" mechanisms. The "cis" mechanism involved translocation-mediated

165 enhancer hijacking in the ETP, TLX and TAL subtypes (Fig. 3d and Supplementary Fig.

$1663 \mathrm{~b}$ ), of which the ectopically expressed genes, such as $T L X 3$, hijacked the enhancers

167 from the translocated BCL11B gene via translocation-mediated loops (Fig. 3e and

168 Supplementary Fig. 3c-d). The "cis" mechanism included BCL11B-TLX3, TRB-TAL2,

169 and 3 novel HOXA translocations identified in this study (Supplementary Fig. 3b and

170 Supplementary Table 6; see Fig. 5). Interestingly, most of the hijacked enhancers are

171 from genes that are normally expressed during T cell development, such as BCL11B

172 and TRB, which lead to ectopic expression of T-ALL-associated transcription factors

173 in the T lineage and block normal differentiation (Supplementary Fig. 3d). The "trans"

174 mechanism involves translocation-mediated gene fusions, such as the PSIP1-NUP98,

175 SET-NUP214, and MLL (KMT2A-MLLT1, PICALM-MLLT10, and DDX3X-MLLT10)

176 gene fusion events, which could epigenetically activate HOXA cluster gene

177 expressions ${ }^{25-28}$. These results suggest that translocation-mediated enhancer

178 hijacking or fusion events may drive ectopic transcription factor activation, leading to

179 specific pathogenic gene expression profiles of the T-ALL subgroups. 
The dysregulated HOXA cluster, which contains 11 genes, is a common feature of $\mathrm{T}$-ALL ${ }^{24}$. However, whether HOXA-positive T-ALLs represent a homogeneous

clinical entity has not been systematically studied. We conducted unsupervised

hierarchical clustering based on HOXA gene expressions, which separated 15 T-ALL

samples without HOXA translocation into HOXA-negative (HOXA) and

HOXA-positive expression $\left(\mathrm{HOXA}^{+}\right)$groups. Translocation-mediated fusion events

could be detected in 5/7 $\mathrm{HOXA}^{+}$samples. The $\mathrm{HOXA}^{+} \mathrm{T}-\mathrm{ALLs}$ could be further

separated into 2 subgroups: the $3^{\prime} \mathrm{HOXA}^{+}$or $5^{\prime} \mathrm{HOXA}^{+}$subgroups, with respect to the

location of the HOXA genes within the HOXA cluster (Fig. 4a). The expression

patterns of the three HOXA translocation cases (HOXA-T, breakpoints shown in Fig.

4b) were closer to those in the $5^{\prime} \mathrm{HOXA}^{+}$subgroup, characterized by ectopic

HOXA11-A13 expressions (Fig. 4a). Interestingly, the $5^{\prime} \mathrm{HOXA}^{+}$and HOXA-T cases in

our Hi-C study are associated with double negative (DN) and ETP phenotypes (Fig.

4a), suggesting that T-ALLs with ectopic HOXA cluster gene expression are

194 heterogeneous entities.

The HOXA genes are transcriptionally repressed in normal $\mathrm{T}$ cells but can be transactivated in T-ALLs by fusion proteins that recruit histone methyltransferase DOT1L to the HOXA locus ${ }^{25,29}$. Although this mechanism uncovered how the HOXA cluster is activated, it cannot explain the diverse HOXA expression patterns associated with different fusion proteins. By integrating $\mathrm{Hi}-\mathrm{C}$ maps with $\mathrm{HOXA}$ gene expression patterns, we found that the differential HOXA gene expressions were associated with different 3D genome organizations in samples without HOXA 
202

204

205

206

207

208

209

210

211

212

213

214

215

216

217

218

219

220

221

222 translocation. Hi-C maps and CTCF motif orientations showed that the 11 HOXA genes were partitioned between 2 TADs (Fig. 4b and Supplementary Fig. 4a): the CTCF binding site C11/13 was used as the $3^{\prime}$ boundary of the $5^{\prime}$ TAD in all samples, while the 5 ' boundaries of the 3' TADs varied among different samples: C7/9 was used by most of the $\mathrm{HOXA}^{-}(6 / 8)$ and all $5^{\prime} \mathrm{HOXA}^{+}$samples (2/2), while $\mathrm{C} 10 / 11$ was used by most of the 3 'HOXA ${ }^{+}$samples (4/5) (red and blue arrows/lines, respectively; Fig. 4b and Supplementary Fig. 4a).

We further identified 6 enhancer regions in each TAD, labeled E1 to E12, which could interact with the HOXA cluster (Fig. 4c). Using HOXA- cases as common denominators (Fig. 4c, top panel), we calculated the overall differential interaction intensities. Although there was no significant difference between the healthy controls and the HOXA cases in the 12 interaction regions, we found significantly enhanced interactions between E2-E6 and genes in the 3'HOXA subgroup, as well as between E8, 9, 11, 12 and genes in the 5'HOXA subgroups, either as a group average (Fig. 4c) or individually (Supplementary Fig. 4b-c). ChIP-seq analysis of the HOXA ${ }^{+}$Loucy cell line indicated that these increased interactions may be correlated with gains in the H3K27ac histone mark (Fig. 4c). The KMT2A-MLLT1, PICALM-MLLT10, DDX3X-MLLT10 and SET-NUP214 fusions were associated with interaction dynamics and HOXA1-A10 expression in the 3'HOXA subgroup, while the PSIP1-NUP98 fusion event was associated with the 5'HOXA subgroup (Fig. 4d). These results suggest that different translocation-mediated fusion events may epigenetically and preferentially 
223

224 from the influence of the hijacked enhancers, leading to 5'HOXA-specific expression

alter the 3D genome interactome within the HOXA cluster and control a specific set of HOXA gene expression.

To explain the gene expression patterns seen in the three HOXA-T samples, we mapped the breakpoints and found that all the breakpoints were located within the $5^{\prime}$ TAD, upstream of the HOXA13 gene. The translocation partner breakpoints lie in the gene bodies of the BCL11B and CDK6 genes on chromosome 14 and chromosome 7, respectively, as well as upstream of the ERG gene on chromosome 21 (Fig. 5 and Supplementary Fig. 5). By examining the TAD structures associated with the translocations, using $\mathrm{HOXA}^{-}$samples as controls, we found that these translocations mediated new loop formations between the 5' of the HOXA cluster and active enhancers associated with BCL11B and ERG genes, leading to ectopic expression of HOXA13 in the case of 076 and HOXA9-A13 in the case of 077 (blue circles for new loops and green bar graphs for gene expressions; Fig. 5a and b; Supplementary Fig. 3b). For the case of 108, the inter-TAD inversion led to the adoption of active CDK6 enhancers and new loop formation (Fig. 5c), causing ectopic expression of HOXA11-A13 (Fig. 5c). These results suggest that translocations and inversion associated with the 5' TAD of the HOXA cluster lead to dysregulated 5' HOXA gene expressions through new loop formation-mediated enhancer hijacking. Again, the existence of the CTCF binding sites C11/13 in the case of 076, C7/9 in the case of 077 , and C10/11 in the case of 108 (Fig. 5d) could insulate genes located in the 3' TAD patterns (Fig. 4b). 
We next investigated whether ectopic HOXA cluster expression was associated

information (see our companion paper). We found that HOXA11 or HOXA13 positivity,

alone or in combination, but not the expression of other HOXA genes, such as the

previously used biomarker HOXA9, was associated with poor overall and event-free

survivals in young adult and pediatric T-ALLs. In contrast, T-ALLs with TAL1/2

positivity, which was mutually exclusive from that with HOXA positivity, were

262 The newly identified translocations and enhancer hijacking mechanism may provide a

263 potential explanation for how T-ALL-associated transcription factors are ectopically 264 activated and block differentiation at certain T cell developmental stages. Finally, we 265 identified the associations of ectopic HOXA11-A13 expression and poor survival of 266 T-ALL, corresponding to the subtype currently incurable by standard treatments, and 
bioRxiv preprint doi: https://doi.org/10.1101/2020.03.11.988279; this version posted March 12, 2020. The copyright holder for this preprint (which was not certified by peer review) is the author/funder, who has granted bioRxiv a license to display the preprint in perpetuity. It is made available under aCC-BY-NC-ND 4.0 International license.

267 suggested that anti-JAK-STAT inhibitors may benefit this group of patients.

268 


\section{Author contributions}

$270 \mathrm{LY}, \mathrm{HZ}$ and HW conceived the project; YC designed the Hi-C and RNA-seq

271 experiments; LY, HZ, MS and WW performed the Hi-C and RNA-seq experiments; FC

272 designed the bioinformatic pipelines and performed the Hi-C and RNA-seq integrated

273 analyses, while BD conducted the survival analysis. QJ, LZ and XH contributed the

274 clinical samples and data. LY, FC and BD generated the figures and tables. LY, FC

275 and HW wrote the manuscript with help from all authors. XH was in charge of the

276 clinical study; MZ and YC oversaw the bioinformatics analyses, and HW supervised

277 the entire project.

278

\section{Acknowledgement}

280 We thank Drs. Meng Lv, Yingjun Chang, and Yan Chang for sample collection; Dr.

281 Cheng Li of Peking University for critically reviewing the manuscript. We also thank

282 Yan Liu, Fei Wang and Xuefang Zhang from the National Center for Protein Sciences

283 Beijing at Peking and Tsinghua Universities for assistance with FACS. This project

284 was supported by the Peking-Tsinghua Center for Life Sciences, Beijing Advanced

285 Innovation Center for Genomics at Peking University for HW and the National Natural

286 Science Foundation of China (81602254 for LY, 31871343 for YC, 31671384 and

28781890994 for YC and MZ). WW was supported by the Postdoctoral Fellowship of

288 Peking-Tsinghua Center for Life Sciences.

289

\section{Conflicts of Interest}

291 The authors declare no competing financial interests. 
293

294

295

296

297

298

299

300

301

302

303

304

305

306

307

308

309

310

311

312

313

314

315

316

317

318

319

320

321

322

\section{Materials and Methods}

Patients and samples. Eighteen T-ALL patient samples were collected under the protocol approved by the ethics committee of the Institute of Hematology at Peking University. The patient characteristics are described in Supplementary Table 7. ETP status was defined as previously published ${ }^{16}$. Leukemia blast cells were prepared by density-gradient centrifugation of bone marrow samples, and CD19-CD14 ${ }^{-} \mathrm{CD} 235^{\circ} \mathrm{CD} 45^{+} \mathrm{CD} 7^{+}$cells were further purified by FACS analysis using anti-human antibodies for RNA-seq and Hi-C library preparations. Peripheral blood samples were obtained from four healthy donors under the approval of the ethics committee of Peking University. T cells were purified using the EasySep ${ }^{\mathrm{TM}}$ Direct Human T Cell Isolation Kit (StemCell Technology \#19661).

\section{RNA-seq library preparation, data processing, and differential gene expression} analysis. RNA-seq libraries were prepared with TruSeq RNA Library Prep Kit v2 (Illumina). Paired-end RNA-seq reads of the 18 patients and 4 healthy controls were generated with an average depth of 15 million read pairs. Reads were aligned to the hg19 genome with TopHat (v2.1.0) using default settings ${ }^{31}$. Duplicates were removed, and aligned reads were calculated for each protein-coding gene using HTSeq ${ }^{32}$, followed by FPKM transformation by normalizing gene exon length and sequencing depth. Raw RNA-seq data for Loucy and Jurkat were downloaded from the GEO database and analyzed as described above.

DESeq $2^{33}$ was applied to identify the differentially expressed genes with FDR $<0.01$ and fold change $>2$. Genes with fewer than 5 reads in $20 \%$ of the samples or with mean reads fewer than 2 were excluded. Signal tracks were generated by using BEDTools ${ }^{34}$ genomeCoveragebed to produce bedGraph files scaled to 1 million reads per data set. Then, the UCSC Genome Browser utility ${ }^{35}$ bedGraphToBigWig was used with default parameters to generate bigwig files.

ChIP-seq, ATAC-seq data processing and motif analysis. ChIP-seq reads were mapped to the hg19 genome with Bowtie $2^{36}$ (v2.3.5) using default parameters, while 
ATAC-seq reads were mapped with Bowtie2 using parameter -X 2000 --no-mixed --no-discordant --no-unal. Aligned reads were filtered for a minimum MAPQ of 20, and duplicates were removed using SAMtools ${ }^{37}$. Signal tracks and peaks were generated by using the -SPMR option in MACS2 ${ }^{38}$. Then, the UCSC Genome Browser utility bedGraphToBigWig was used with default parameters to transform the bedgraph files to bigwig files. $\mathrm{FIMO}^{39}$ was used to detect the $20 \mathrm{bp}$ CTCF motif from the Homer motif database in Loucy and Jurkat CTCF peaks with default parameters.

$\mathrm{Hi}-\mathrm{C}$ and Hi-C data processing. Hi-C was performed on one million cells/sample, according to the $\mathrm{BL}-\mathrm{Hi}-\mathrm{C}$ protocol ${ }^{13}$. Raw $\mathrm{BL}-\mathrm{Hi}-\mathrm{C}$ reads were processed by the in-house HiCpipe framework, which integrated several $\mathrm{Hi}-\mathrm{C}$ analysis methods for to generate multiple features of the Hi-C data. In particular, ChIA-PET2 ${ }^{40}$ was used to trim the bridge linkers and $\mathrm{HiC}-\mathrm{PrO}^{41}$ to align reads, filter artifact fragments, and remove duplicates; Juicer ${ }^{42}$ was applied to the resulting uniquely mapped contacts to generate individual or merged $\mathrm{Hi}-\mathrm{C}$ files that could be deposited as contact matrices with multiple resolutions. Knight-Ruiz ${ }^{43}(K R)$-normalized matrices were used in the compartment and TAD analyses.

Compartment and TAD analysis. The compartment was calculated with the eigenvector command of Juicer under $100 \mathrm{~kb}$ resolution $\mathrm{KR}$ normalized $\mathrm{Hi}-\mathrm{C}$ matrices. For every $100 \mathrm{~kb}$ bin, A or B compartments were defined by the over $70 \%$ sample majority rule.

TAD boundaries were calculated by the Insulation score method ${ }^{44}$ (with parameters: -is 1000000 -ids 200000 -im mean -nt 0.1) on pooled $40 \mathrm{~kb} \mathrm{Hi}-\mathrm{C}$ matrices of the healthy T cell controls, ETP and non-ETP samples. The resulting TAD boundaries were merged and assigned with relative insulation scores of all samples calculated from $\mathrm{HiCDB}^{45}$. Differential TAD boundaries were defined with a t-test FDR $<0.01$ and a difference between cases and controls higher than $50 \%$ quantile of the overall difference.

A TAD was defined when its boundaries were detected in at least two conditions 
among normal T cells, ETPs and non-ETPs. The domain score ${ }^{46}$ was calculated in each sample by dividing the intra-TAD interactions with all interactions connected to the corresponding TAD. Differential domain scores were calculated with a t-test FDR $<0.01$ and fold change higher than $70 \%$ quantile of the overall fold change.

Loop detection and differential loop calling. Loops were called by HiCCUPS ${ }^{42}$ at 5 $\mathrm{kb}$ and $10 \mathrm{~kb}$ resolutions with default parameters (except -d 15000,20000) for pooled $\mathrm{Hi}-\mathrm{C}$ matrices of the healthy T cell controls, ETP and non-ETP samples, respectively. The differential loop detection method was adapted from Douglas et $a l^{47}$. Loops were split into two distance ranges ( $>$ or $<150 \mathrm{~kb}$ ) to minimize potential bias (Rubin et al. ${ }^{48}$ ). Differential loops were called within each range $($ FDR $<0.1)$ and then combined.

Loop aggregation and functional analysis. Aggregate peak analysis (APA) plots were generated to assess the quality of loop detection and explore the characteristics of different loop classes by the Juicer APA command ${ }^{42}$ under $5 \mathrm{~kb}$ resolution. Its output matrix was normalized by the loop number that contributed to the matrix generation. For analysis of the function of dynamic loops between non-ETP and ETP, the loop anchors were analyzed by GREAT ${ }^{49}$ (v3.0.0) using the nearest gene within $100 \mathrm{~kb}$ to generate the enriched biological process. In other sections, genes related to loops were determined if their promoter ( $5 \mathrm{~kb}$ around TSS) overlapped the loop anchors, and DAVID $^{50} 6.8$ was used for KEGG pathway enrichment analysis.

Visualization and V4C plot generation. Tracks of Hi-C maps and ChIP-seq were generated by pyGenomeTracks ${ }^{51}$. Hi-C maps of each condition were normalized by its cis interaction pairs. A visual 4C (V4C) plot for specific loci was generated as the interaction s related to the corresponding viewpoint under $10 \mathrm{~kb}$ resolution.

\section{Translocation and translocation-mediated loop detection with $\mathrm{Hi}-\mathrm{C}$.} hic_breakfinder ${ }^{20}$ was adapted to detect translocations in 18 T-ALL patient samples. After we filtered the "translocations" also detected in normal controls, the remaining 
translocations were manually assessed, and the precise breakpoints were determined. As the average depth of patient $\mathrm{Hi}-\mathrm{C}$ samples is 486 million read pairs and the read length is $150 \mathrm{bp}, \mathrm{Hi}-\mathrm{C}$ raw data were treated as single ends to refine the breakpoint locations to single base-pair resolution. Any single ends that could be mapped to two different chromatins without the BL-Hi-C bridge linker in between were chimeric reads. The chimeric reads detected from the BL-Hi-C data overlapped with the aforementioned translocations at $5 \sim 20 \mathrm{~kb}$ resolution. For each translocation, the exact locations (single base-pair resolution) supported by more than 3 chimeric reads were identified as the actual breakpoints and are reported in Supplementary Table 5.

As translocation-mediated loops were hard to identify by loop detection tools designed for intrachromosomal loop detection and easy to capture by visualization, their locations were manually recorded on interchromosome Hi-C maps with the help of Juicebox ${ }^{52}$, which is an interactive visualization software.

Translocation, translocation-mediated loop annotation and visualization. The nearest genes to translocation breakpoints were determined by BEDTools. Known translocations were collected from references 2 and 4 and ChimerPub ${ }^{53}$. A translocation was considered novel if any of the breakpoints was not near any known breakpoint within a $100 \mathrm{~kb}$ distance. For translocation-mediated loops, the genes with a promoter or gene body overlapping the loop anchors were annotated as the associated genes. A gene near a breakpoint was considered upregulated if its FPKM was $>1$ - and 2-fold higher than the control samples without nearby breakpoints. Hi-C heatmaps and Visual 4C plots of the reassembled chromatin were generated by MATLAB code.

Statistics. Specific statistical analyses are described in each section. In general, the Wilcoxon rank sum test was employed in $\mathrm{R}$ for comparisons of distributions. Survival analysis was performed by a Cox regression model using overall and event-free survival as outcomes. Overall survival was defined as the time from diagnosis to death from any cause. Event-free survival was defined as the time from diagnosis to treatment 
413 failure, relapse, or death from any cause. The proportional hazard assumption was

414 tested. Variables tested in the multivariable Cox regression model were sex, age

415 (pediatric vs. adult), white blood cell counts, hemoglobin levels, platelet counts, 416 hepatosplenomegaly, percentage of blasts in the bone marrow and MRD status.

417 Eighty-six patient samples with RNA-seq data were used for DN- and ETP-

418 enrichment analyses, of which 38 samples under the age of 40 with outcome and 63

419 samples with whole exon sequencing (WES) data were used for survival and mutation

420 analyses, respectively.

421

422 Code availability. Custom scripts described in the Online Methods will be made 423 available upon request.

424

425 Data availability. All sequencing data are available through the Gene Expression 426 Omnibus (GEO) via accession GSE. Accession codes of the published data used in 427 this study are as follows: CTCF ChIP-seq of CD4+ $T$ cell and Jurkat cell line, 428 GSE12889; CTCF ChIP-seq of Loucy cell line, GSE123214; ATAC-seq of CD4+ T cell, 429 GSE87254; ATAC-seq of Jurkat cell line, GSE115438; H3K27ac ChIP-seq of CD4+ T 430 cell, GSE122826; H3K27ac ChIP-seq of Jurkat cell line, GSE68978; H3K27ac 431 ChIP-seq of Loucy cell line, GSE74311; RNA-Seq of Loucy cell line, GSE100694; 432 RNA-seq of T cell development, GSE69239. The raw sequence data reported in this 433 paper have been deposited in the Genome Sequence Archive ${ }^{54}$ in BIG Data Center ${ }^{55}$, 434 Beijing Institute of Genomics (BIG), Chinese Academy of Sciences, under accession 435 number HRA000113 that is publicly accessible at https://bigd.big.ac.cn/gsa. 
439 References

$4401 \quad$ Orlando, G. et al. Promoter capture Hi-C-based identification of recurrent noncoding

441 mutations in colorectal cancer. Nat Genet 50, 1375-1380,

442 doi:10.1038/s41588-018-0211-z (2018).

4432 Flavahan, W. A. et al. Insulator dysfunction and oncogene activation in IDH mutant

444 gliomas. Nature 529, 110-114, doi:10.1038/nature16490 (2016).

4453 Yu, M. \& Ren, B. The Three-Dimensional Organization of Mammalian Genomes. Annu

446 Rev Cell Dev Bio/33, 265-289, doi:10.1146/annurev-cellbio-100616-060531 (2017).

4474 Groschel, S. et al. A single oncogenic enhancer rearrangement causes concomitant

448 EVI1 and GATA2 deregulation in leukemia. Cell 157, 369-381,

449 doi:10.1016/j.cell.2014.02.019 (2014).

4505 Hnisz, D. et al. Activation of proto-oncogenes by disruption of chromosome

451 neighborhoods. Science 351, 1454-1458, doi:10.1126/science.aad9024 (2016).

4526 Petrovic, J. et al. Oncogenic Notch Promotes Long-Range Regulatory Interactions

453 within Hyperconnected 3D Cliques. Mol Cell 73, 1174-1190 e1112,

454 doi:10.1016/j.molcel.2019.01.006 (2019).

4557 Kloetgen, A., Thandapani, P., Tsirigos, A. \& Aifantis, I. 3D Chromosomal Landscapes

456 in Hematopoiesis and Immunity. Trends Immunol 40, 809-824,

457 doi:10.1016/j.it.2019.07.003 (2019).

$458 \quad 8 \quad$ Belver, L. \& Ferrando, A. The genetics and mechanisms of T cell acute lymphoblastic

459 leukaemia. Nat Rev Cancer 16, 494-507, doi:10.1038/nrc.2016.63 (2016).

4609 Liu, Y. et al. The genomic landscape of pediatric and young adult T-lineage acute 
lymphoblastic leukemia. Nat Genet 49, 1211-1218, doi:10.1038/ng.3909 (2017). lymphoblastic leukemia. Nat Genet 49, 1274-1281, doi:10.1038/ng.3900 (2017).

46411 Chen, B. et al. Identification of fusion genes and characterization of transcriptome features in T-cell acute lymphoblastic leukemia. Proc Natl Acad Sci U S A 115, 373-378, doi:10.1073/pnas.1717125115 (2018). leukaemia. Nature 481, 157-163, doi:10.1038/nature10725 (2012).

Liang, Z. et al. BL-Hi-C is an efficient and sensitive approach for capturing structural and regulatory chromatin interactions. Nat Commun 8, 1622, doi:10.1038/s41467-017-01754-3 (2017). leukemia. Cancer Cel/22, 452-465, doi:10.1016/j.ccr.2012.09.016 (2012).

47415 Aibar, S. et al. SCENIC: single-cell regulatory network inference and clustering. Nat Methods 14, 1083-1086, doi:10.1038/nmeth.4463 (2017).

$47616 \quad$ Coustan-Smith, E. et al. Early T-cell precursor leukaemia: a subtype of very high-risk acute lymphoblastic leukaemia. Lancet Oncol 10, 147-156, doi:10.1016/S1470-2045(08)70314-0 (2009).

$47917 \mathrm{Hu}, \mathrm{G}$. et al. Transformation of Accessible Chromatin and 3D Nucleome Underlies Lineage Commitment of Early $\mathrm{T}$ Cells. Immunity 48, 227-242 e228, doi:10.1016/j.immuni.2018.01.013 (2018). 
483

484

485

486

487

$488 \quad 20$

489

490

491

493

494

495

496

497

498

499

500

501

$502 \quad 26$

503

504 reveals transcriptional divergence of B cell and T cell lineages. Nat Immuno/ 16 ,

1282-1291, doi:10.1038/ni.3299 (2015).

19 Lee, J. K., Choi, Y. L., Kwon, M. \& Park, P. J. Mechanisms and Consequences of Cancer Genome Instability: Lessons from Genome Sequencing Studies. Annu Rev Pathol-Mech 11, 283-312, doi:10.1146/annurev-pathol-012615-044446 (2016).

20 Dixon, J. R. et al. Integrative detection and analysis of structural variation in cancer genomes. Nat Genet 50, 1388-1398, doi:10.1038/s41588-018-0195-8 (2018).

21 Fudenberg, G. et al. Formation of Chromosomal Domains by Loop Extrusion. Cell Rep 15, 2038-2049, doi:10.1016/j.celrep.2016.04.085 (2016).

22 Nuebler, J., Fudenberg, G., Imakaev, M., Abdennur, N. \& Mirny, L. A. Chromatin organization by an interplay of loop extrusion and compartmental segregation. Proc Natl Acad Sci U S A 115, E6697-E6706, doi:10.1073/pnas.1717730115 (2018).

23 Davidson, I. F. et al. DNA loop extrusion by human cohesin. Science 366, 1338-1345, doi:10.1126/science.aaz3418 (2019).

24 Soulier, J. et al. HOXA genes are included in genetic and biologic networks defining human acute T-cell leukemia (T-ALL). Blood 106, 274-286, doi:10.1182/blood-2004-10-3900 (2005).

25 Bernt, K. M. et al. MLL-rearranged leukemia is dependent on aberrant H3K79 methylation by DOT1L. Cancer Cel/20, 66-78, doi:10.1016/j.ccr.2011.06.010 (2011).

26 Jo, S. Y., Granowicz, E. M., Maillard, I., Thomas, D. \& Hess, J. L. Requirement for Dot1l in murine postnatal hematopoiesis and leukemogenesis by MLL translocation. Blood 117, 4759-4768, doi:10.1182/blood-2010-12-327668 (2011). 
50527 Nguyen, A. T., Taranova, O., He, J. \& Zhang, Y. DOT1L, the H3K79 methyltransferase,

506 is required for MLL-AF9-mediated leukemogenesis. Blood 117, 6912-6922,

507 doi:10.1182/blood-2011-02-334359 (2011).

50828 Okada, Y. et al. hDOT1L links histone methylation to leukemogenesis. Cell 121, $509 \quad$ 167-178, doi:10.1016/j.cell.2005.02.020 (2005).

51029 Barry, E. R., Corry, G. N. \& Rasmussen, T. P. Targeting DOT1L action and 511 interactions in leukemia: the role of DOT1L in transformation and development. Expert $512 \quad$ Opin Ther Targets 14, 405-418, doi:10.1517/14728221003623241 (2010).

51330 de Bock, C. E. et al. HOXA9 Cooperates with Activated JAK/STAT Signaling to Drive 514 Leukemia Development. Cancer Discov 8, 616-631, 515 doi:10.1158/2159-8290.CD-17-0583 (2018).

51631 Trapnell, C. et al. Differential gene and transcript expression analysis of RNA-seq 517 experiments with TopHat and Cufflinks. Nat Protoc 7, 562-578, 518 doi:10.1038/nprot.2012.016 (2012).

51932 Anders, S., Pyl, P. T. \& Huber, W. HTSeq--a Python framework to work with 520 high-throughput sequencing data. Bioinformatics 31, 166-169, 521 doi:10.1093/bioinformatics/btu638 (2015).

52233 Love, M. I., Huber, W. \& Anders, S. Moderated estimation of fold change and 523 dispersion for RNA-seq data with DESeq2. Genome Biol 15, 550, 524 doi:10.1186/s13059-014-0550-8 (2014).

52534 Quinlan, A. R. \& Hall, I. M. BEDTools: a flexible suite of utilities for comparing genomic 526 features. Bioinformatics 26, 841-842, doi:10.1093/bioinformatics/btq033 (2010). 
$52735 \quad$ Kuhn, R. M., Haussler, D. \& Kent, W. J. The UCSC genome browser and associated

528 tools. Brief Bioinform 14, 144-161, doi:10.1093/bib/bbs038 (2013).

$52936 \quad$ Langmead, B. \& Salzberg, S. L. Fast gapped-read alignment with Bowtie 2. Nat $530 \quad$ Methods 9, 357-359, doi:10.1038/nmeth.1923 (2012).

$53137 \quad \mathrm{Li}, \mathrm{H}$. et al. The Sequence Alignment/Map format and SAMtools. Bioinformatics 25, $532 \quad$ 2078-2079, doi:10.1093/bioinformatics/btp352 (2009).

$533 \quad 38$ Zhang, Y. et al. Model-based analysis of ChIP-Seq (MACS). Genome Biol 9, R137, doi:10.1186/gb-2008-9-9-r137 (2008).

53539 Grant, C. E., Bailey, T. L. \& Noble, W. S. FIMO: scanning for occurrences of a given 536 motif. Bioinformatics 27, 1017-1018, doi:10.1093/bioinformatics/btr064 (2011).

53740 Li, G., Chen, Y., Snyder, M. P. \& Zhang, M. Q. ChIA-PET2: a versatile and flexible pipeline for ChIA-PET data analysis. Nucleic Acids Res 45, e4, doi:10.1093/nar/gkw809 (2017).

54041 Servant, N. et al. HiC-Pro: an optimized and flexible pipeline for $\mathrm{Hi}-\mathrm{C}$ data processing. Genome Bio/ 16, 259, doi:10.1186/s13059-015-0831-x (2015).

54242 Rao, S. S. et al. A 3D map of the human genome at kilobase resolution reveals 543 principles of chromatin looping. Cell 159, 1665-1680, doi:10.1016/j.cell.2014.11.021 $544 \quad$ (2014).

54543 Knight, P. A. \& Ruiz, D. A fast algorithm for matrix balancing. Ima J Numer Ana/ 33, $546 \quad$ 1029-1047, doi:10.1093/imanum/drs019 (2013).

54744 Giorgetti, L. et al. Structural organization of the inactive $X$ chromosome in the mouse. 
54945 Chen, F., Li, G., Zhang, M. Q. \& Chen, Y. HiCDB: a sensitive and robust method for 550 detecting contact domain boundaries. Nucleic Acids Res 46, 11239-11250, $551 \quad$ doi:10.1093/nar/gky789 (2018).

$55246 \quad$ Stadhouders, R. et al. Transcription factors orchestrate dynamic interplay between 553 genome topology and gene regulation during cell reprogramming. Nat Genet 50, $554 \quad$ 238-249, doi:10.1038/s41588-017-0030-7 (2018).

55547 Phanstiel, D. H. et al. Static and Dynamic DNA Loops form AP-1-Bound Activation 556 Hubs during Macrophage Development. Mol Cell 67, 1037-1048 e1036, 557 doi:10.1016/j.molcel.2017.08.006 (2017).

$55848 \quad$ Rubin, A. J. et al. Lineage-specific dynamic and pre-established enhancer-promoter 559 contacts cooperate in terminal differentiation. Nat Genet 49, 1522-1528, doi:10.1038/ng.3935 (2017).

56149 McLean, C. Y. et al. GREAT improves functional interpretation of cis-regulatory 562 regions. Nat Biotechno/ 28, 495-501, doi:10.1038/nbt.1630 (2010).

$56350 \quad$ Huang da, W., Sherman, B. T. \& Lempicki, R. A. Systematic and integrative analysis 564 of large gene lists using DAVID bioinformatics resources. Nat Protoc 4, 44-57, 565 doi:10.1038/nprot.2008.211(2009).

$56651 \quad$ Ramirez, F. et al. High-resolution TADs reveal DNA sequences underlying genome 567 organization in flies. Nat Commun 9, 189, doi:10.1038/s41467-017-02525-w (2018).

56852 Durand, N. C. et al. Juicebox Provides a Visualization System for Hi-C Contact Maps 569 with Unlimited Zoom. Cell Syst 3, 99-101, doi:10.1016/j.cels.2015.07.012 (2016).

$57053 \quad$ Kim, P. et al. ChimerDB 2.0--a knowledgebase for fusion genes updated. Nucleic 

available under aCC-BY-NC-ND 4.0 International license.

571

57254 Wang, Y. et al. GSA: Genome Sequence Archive<sup/>. Genomics Proteomics

573

$57455 \quad$ National Genomics Data Center, M. \& Partners. Database Resources of the National

Genomics

Data

Center in 2020.

Nucleic

Acids

Res

48, D24-D33,

576

doi:10.1093/nar/gkz913 (2020).

577 


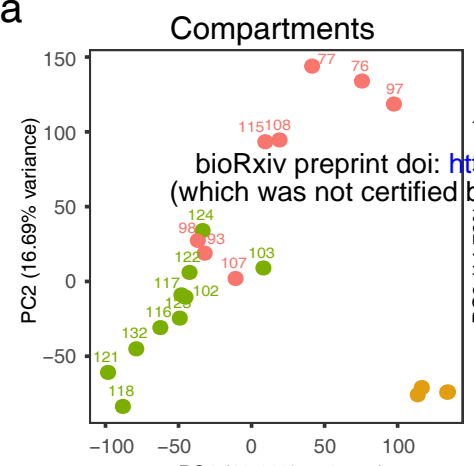

D-score

(tins://doi.org/310.1

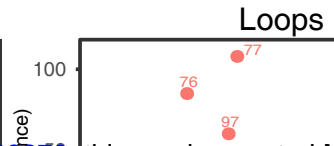

Loops

b

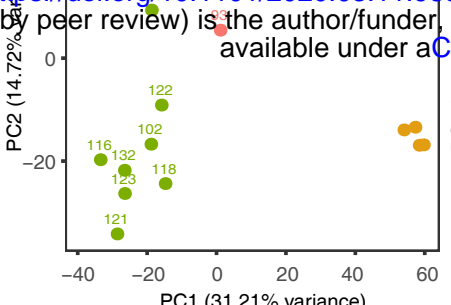

79 ; this version

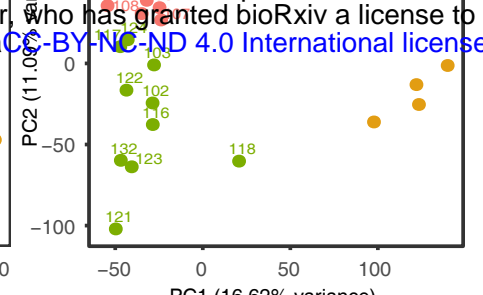

PC1 $(16.62 \%$ variance)

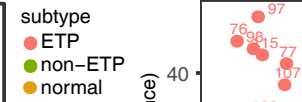
PC1 (19.39\% variance)

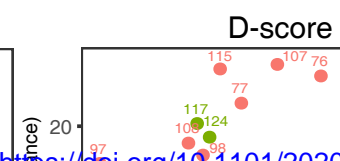

$101 / 20200311$ बे

970

RNA

2020. The

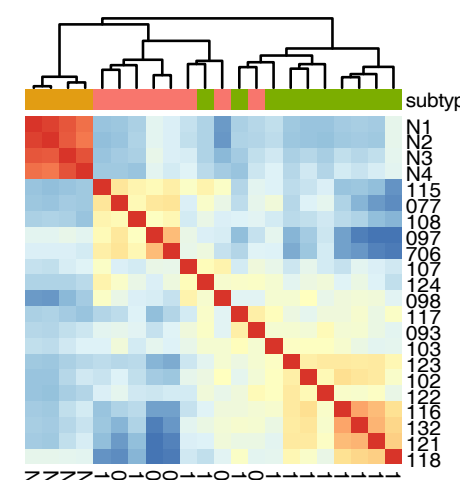

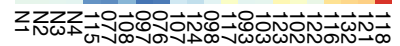

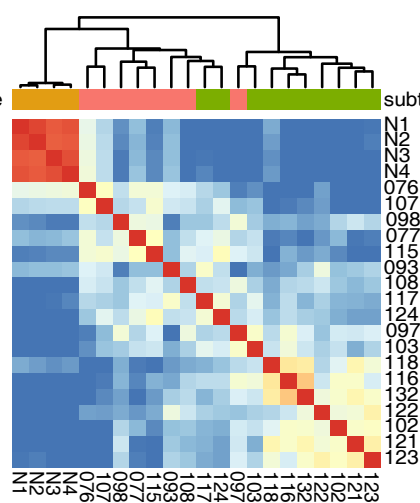

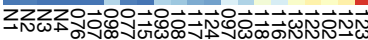

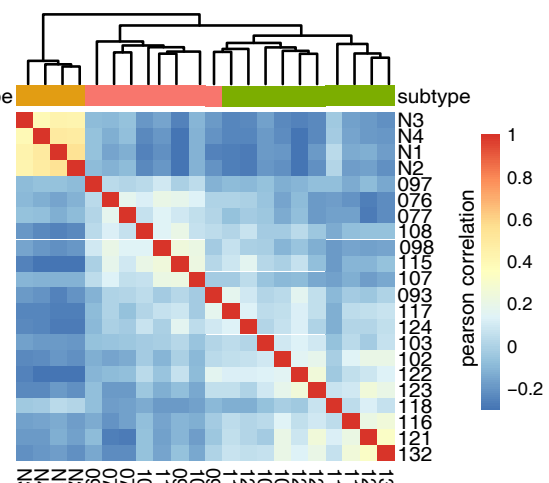

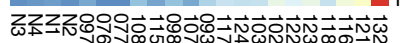

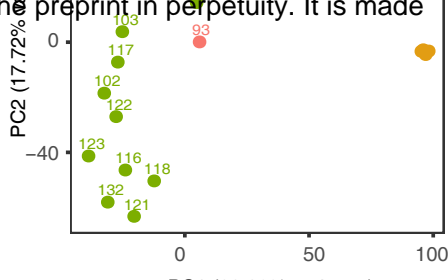

PC1 (28.30\% variance)
C

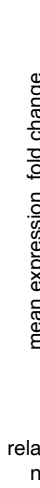

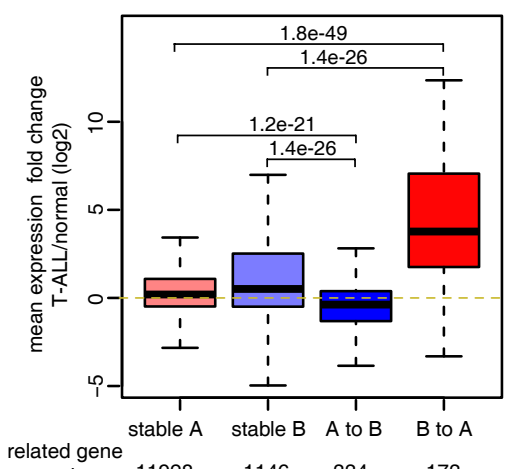

e

pathway of T-ALL enhanced structure related DEGs hematopoietic cell lineage nal misregulation in cancer cell cycle pathways in cancer signaling pathways regulating pluripotency of stem cells

$-\log _{10}(P$-value $)$

pathway of normal enhanced structure related DEGs

cytokine-cytokine receptor interaction $\begin{array}{lllll}0 & 2 & 4 & 6 & 8\end{array}$

$T$ cell receptor signaling pathway endocytosis Jak-STAT signaling pathway

Jak-STAT signaling pathway
axon guidance 0246

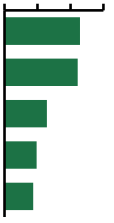
$-\log _{10}(P$-value $)$

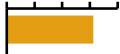

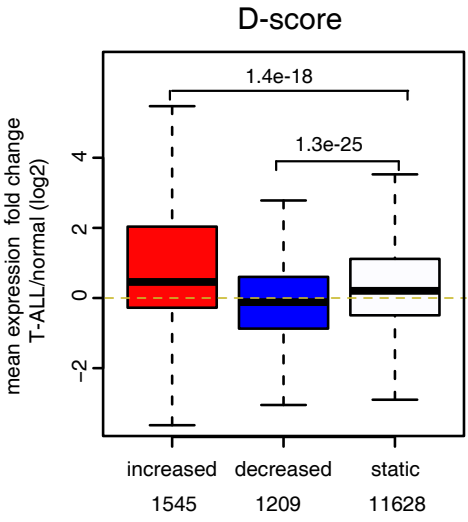

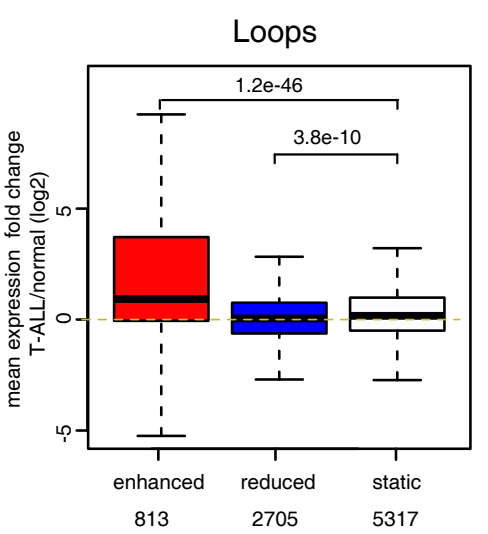

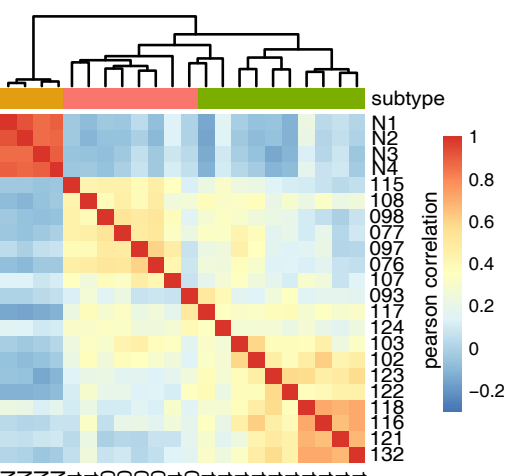

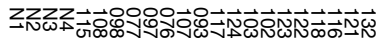

d

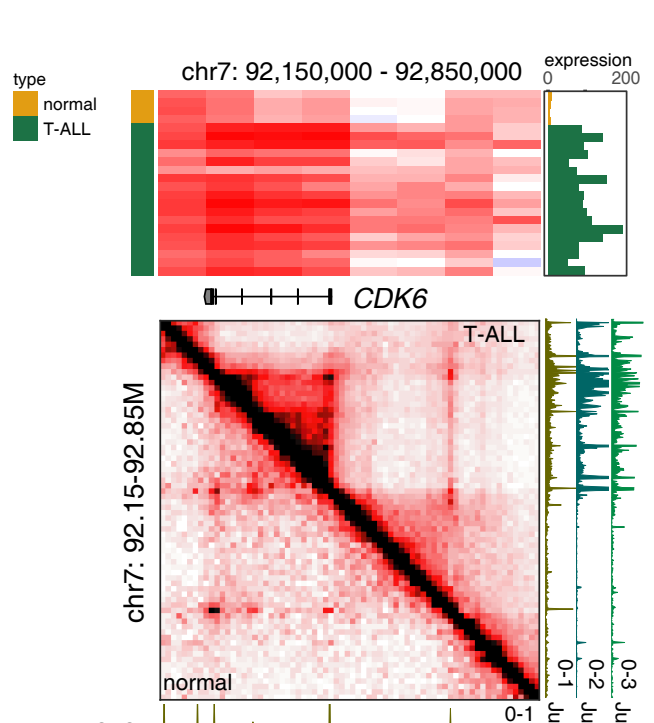

g
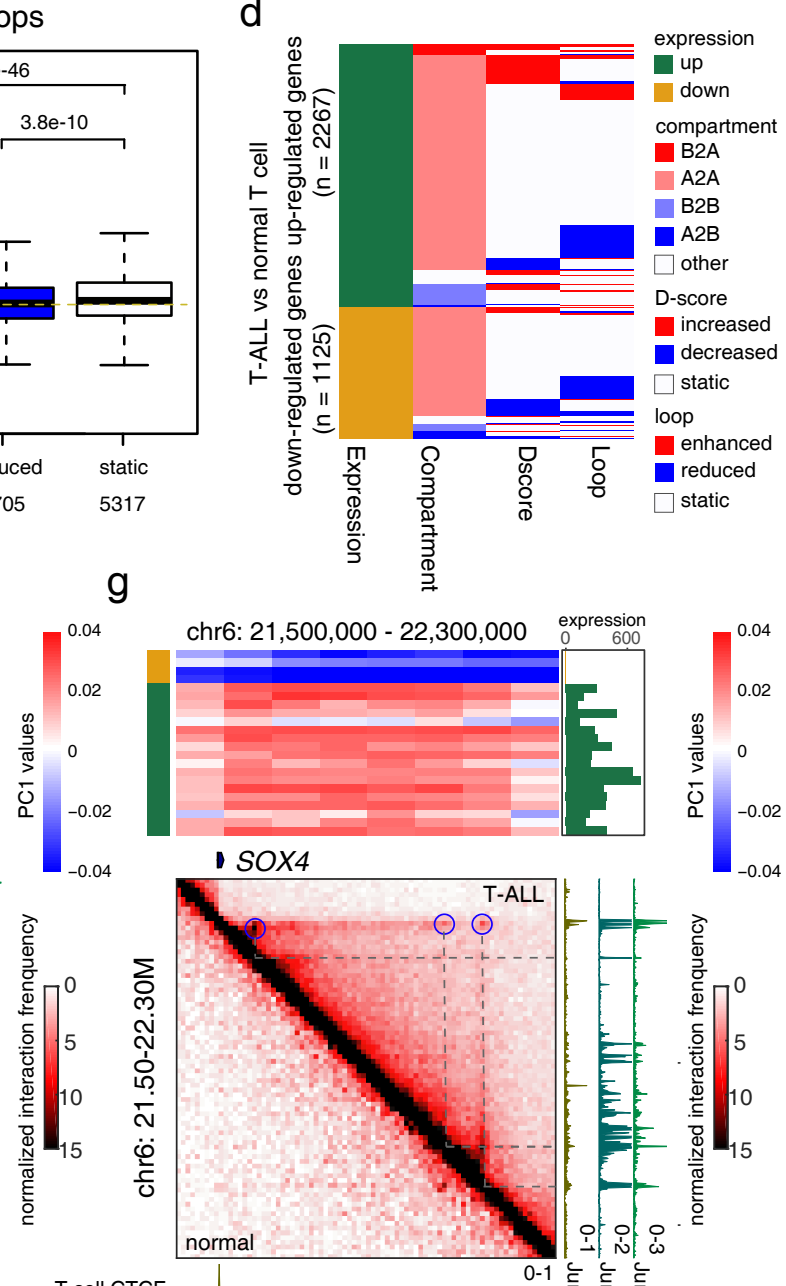

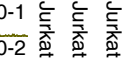

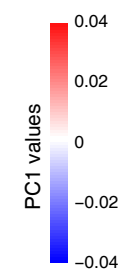

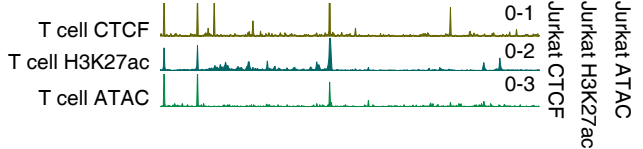

T cell CTCF
T cell H3K27ac
T cell ATAC

$0-3$ 号壱 
bioRxiv preprint doi: https://doi.org/10.1101/2020.03.11.988279; this version posted March 12, 2020. The copyright holder for this preprint (which was not certified by peer review) is the author/funder, who has granted bioRxiv a license to display the preprint in perpetuity. It is made Fig.1 Global 3D genome architectures in T-ALLs. (a) PCA (upper) and unsupervised hierarchical clustering analysis (lower) of compartment, domain score (D-score) and loop in normal T cells and T-ALLs. (b) PCA (upper) and unsupervised hierarchical clustering analysis (lower) of gene expression profiles in normal T cells and T-ALLs. (c) Association of DEGs and genomic alterations at the levels of compartment, TAD and loop. Red, upregulation; blue, downregulated. The $\mathrm{p}$ values were calculated using Wilcoxon rank sum test. (d) A summary of DEGs and their corresponding chromatin structure changes. (e) KEGG analysis for enriched pathways in T-ALLs and normal T cells based on DEGs that are associated with chromatin structural changes. (f) and (g) Top, heatmaps show the compartment scores for each sample across the genomic regions of the CDK6 and SOX4 loci, respectively. Bar plots on the right show the gene expression level of CDK6 and SOX4 for each sample. Bottom, Hi-C contact maps of the CDK6 and SOX4 loci in normal T cell and T-ALL; blue circles: enhanced loops in T-ALL. ATAC-seq tracks and ChIP-seq tracks of CTCF and H3K27ac of normal T cells and T-ALL Jurket cells are also included. 


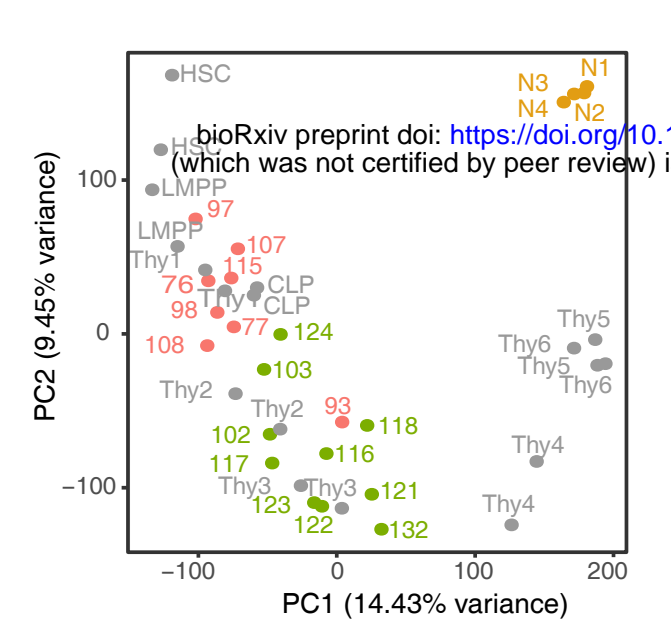

- development • ETP • non-ETP • normal

\section{d}

GO terms of ETP enhanced loop related genes

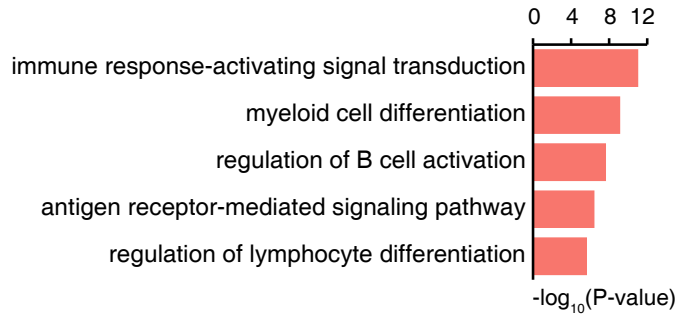

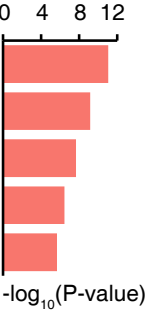

GO terms of non-ETP enhanced loop related genes

$\begin{array}{rrrr}0 & 4 & 8 & 12\end{array}$

positive regulation of RNA metabolic process

positive regulation of transcription, DNA-dependent

leukocyte differentiation

hematopoietic or lymphoid organ development

$T$ cell receptor $V(D) J$ recombination

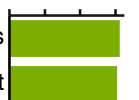

\section{की}

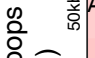

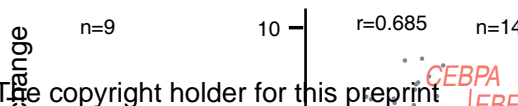

althor/funder who has ganted bioRxiv a license to display the preprint in perpetulty. It is made. EBF viluble under aCC-BY-NC-ND 4.0 International license.
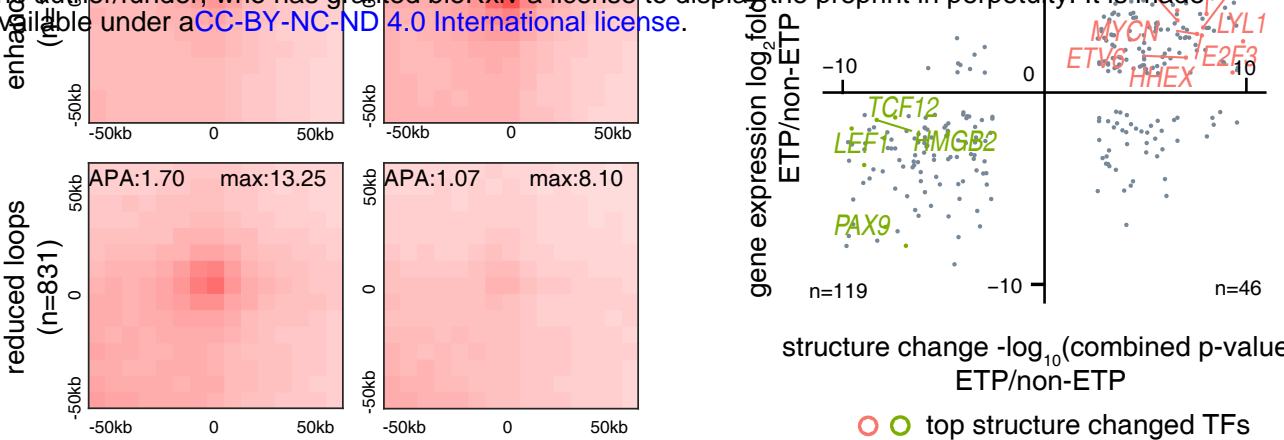

structure change - $\log _{10}$ (combined $p$-value) ETP/non-ETP

- top structure changed TFs

e
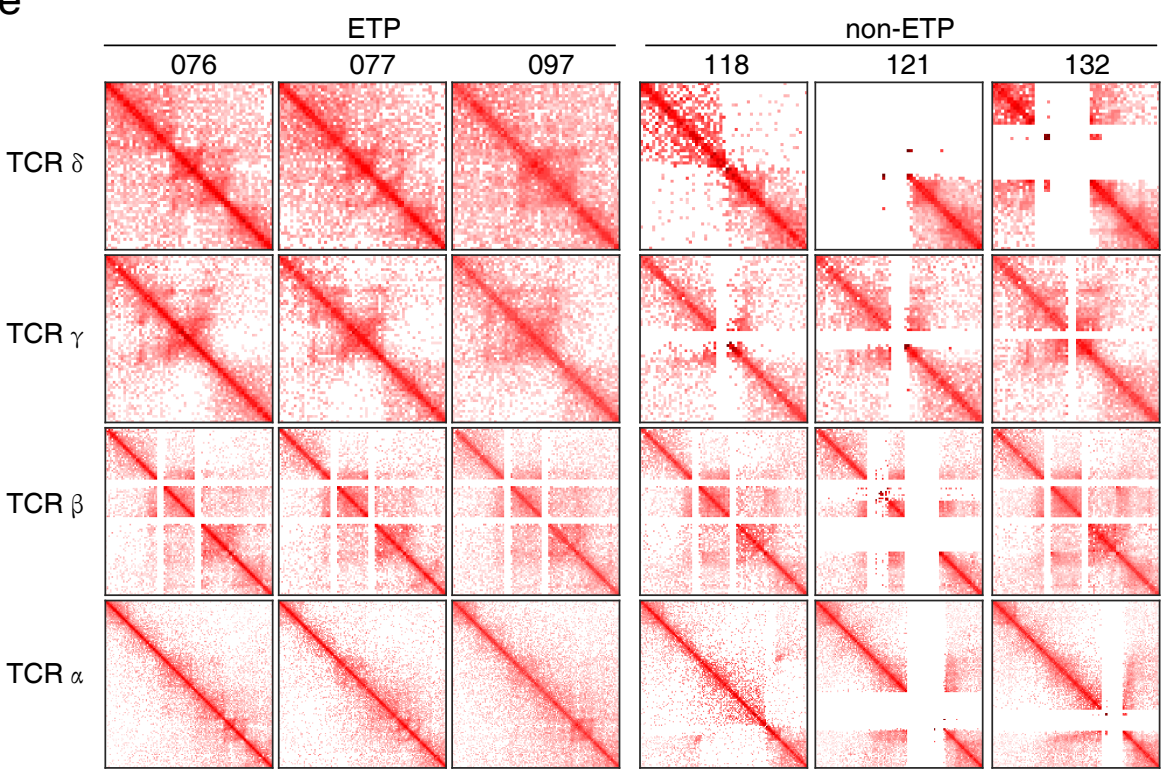

g chr5: $87,950,000-88,250,000$

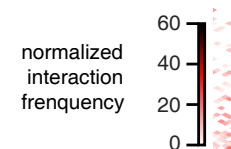

RNA expression

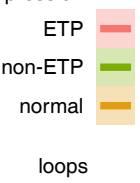

ETP enhanced non-ETP enhanced

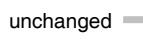

RNAseq

T cell CTCF

Jurkat CTCF

T cell H3K27ac

Jurkat H3K27ac

KE37 H3K27ac

Hi-C loops

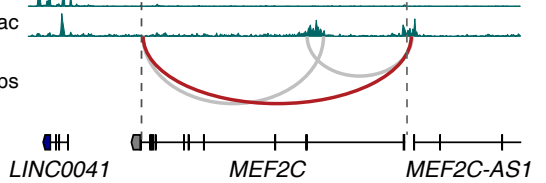

h

chr14: $36,900,000-37,700,000$

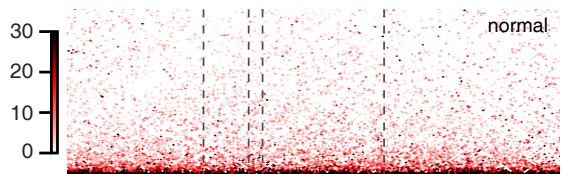

non-ETP

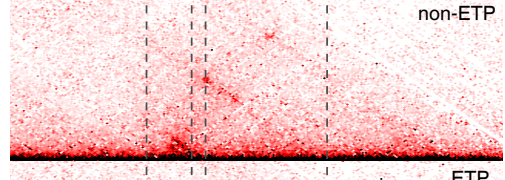

ETP
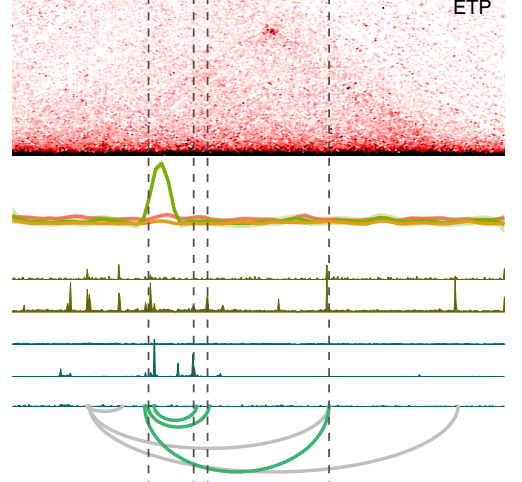

I I H I NKX2-1 PAX9 MIR4503 
bioRxiv preprint doi: https://doi.org/10.1101/2020.03.11.988279; this version posted March 12, 2020. The copyright holder for this preprint (which was not certified by peer review) is the author/funder, who has granted bioRxiv a license to display the preprint in perpetuity. It is made Fig. 2 ETP and non-ETP ALLs have different loop structures. (a) PCA shows the association between ETP and non-ETP ALL gene expression profiles and T cell developmental trajectory. (b) APA plots for loops that are enhanced (top) or reduced (bottom) in ETP compared with non-ETP ALL. (c) Scatterplot shows the correlation between gene expression changes and structural changes. The structural change was defined by the combined $p$-value of D-score and loop strength change. Top ETP and non-ETP ALL-associated transcription factors with structural changes are highlighted in red and green, respectively. (d) GO terms for genes adjacent to enhanced loop anchors in ETP (top) and non-ETP (bottom). (e) Hi-C contact maps for the TCR genomic regions in ETP and non-ETP ALLs. (f) RAG1 and PTCRA expression levels. (g) and (h) Hi-C contact maps for TADs enclosing the genomic loci of ETP expressed MEF2C (g) and non-ETP expressed PAX9 (h). Anchors of differential loops are labeled with dashed lines. 


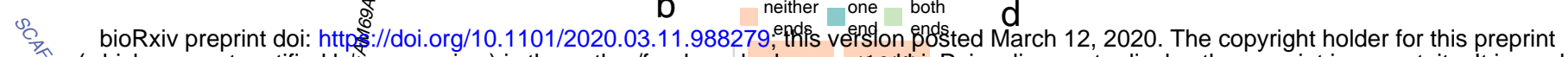
(which was not certified by peer review) is the author/funder ${ }_{40}$ wha

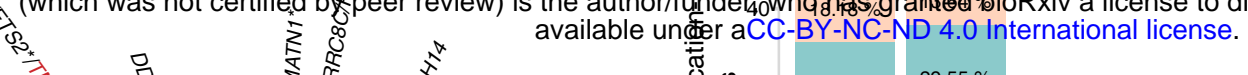
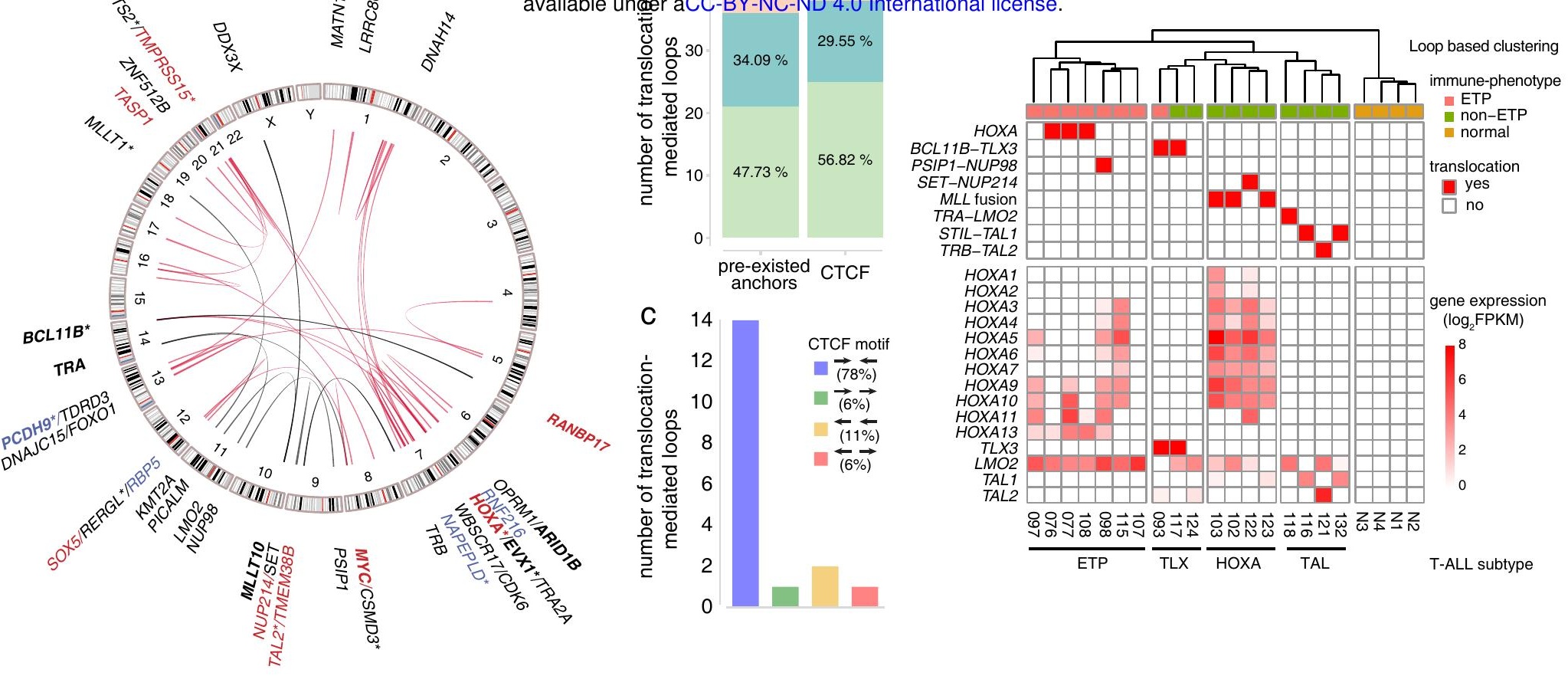

e
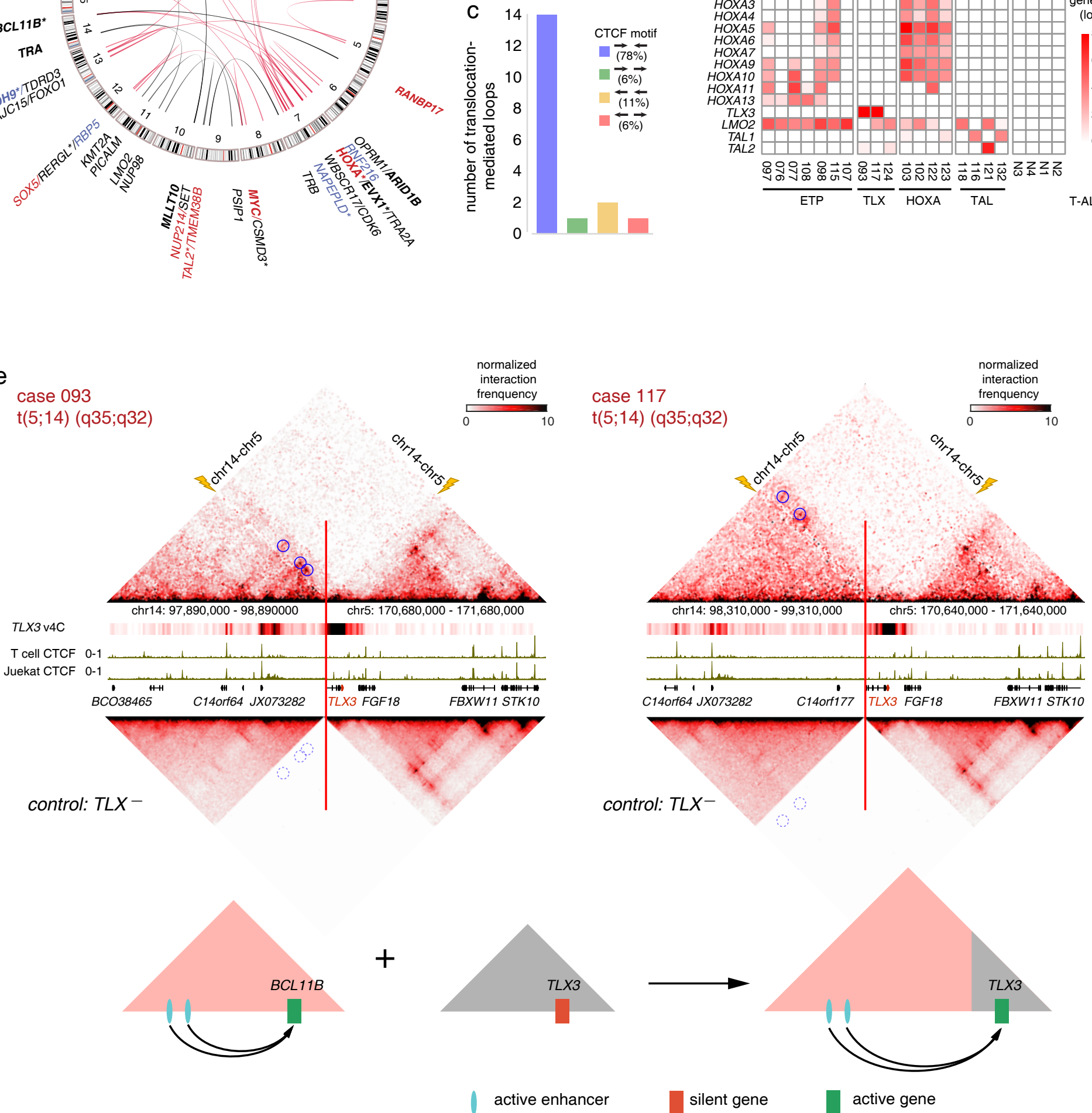

active enhancer

silent gene 
bioRxiv preprint doi: https://doi.org/10.1101/2020.03.11.988279; this version posted March 12, 2020. The copyright holder for this preprint (which was not certified by peer review) is the author/funder, who has granted bioRxiv a license to display the preprint in perpetuity. It is made Fig. 3 Chromosomal rearrangements in T-ALLS. (a) The genomic landscape of translocations discovered by $\mathrm{Hi}-\mathrm{C}$. The novel translocation partners are connected by red lines and known translocations are shown by black lines. Breakpoints nearest genes with increased or decreased expressions are highlighted by red and blue, respectively. Recurrent breakpoints nearest genes are labeled in bold. The genes closest to the breakpoint in noncoding regions are marked by star symbols. (b) Majority of the translocation-mediated loop anchors are pre-existing loop anchors and contains CTCF binding sites. (c) Majority of translocation-mediated loops have convergent CTCF motif orientation. (d) The loop-based clustering overlaps with leukemogenic transcription factor- or translocations-based clustering in T-ALLS. STIL-TAL1 fusions were detected by RT-qPCR, the other translocations were detected by Hi-C. (e) Upper panels: Hi-C heatmaps for cases with BCL11B-TLX3 translocation (top), visual 4C plots generated from $\mathrm{Hi}-\mathrm{C}$ contact maps using $T L X 3$ promoter as the viewpoint (middle) and averaged $\mathrm{Hi}-\mathrm{C}$ heatmaps for cases without BCL11B-TLX3 translocation as controls (bottom). Breakpoints are marked by the red lines and yellow lightning bolts. Translocation-mediated loops are highlighted by blue circles, corresponding loop locations at controls are highlighted by dotted line circles. Lower panels: a schematic illustration for the consequence of the BCL11B-TLX3 translocation. 
a

CD4/CD8 phenotype b

chr7 $27.1 \quad$ HOXA cluster

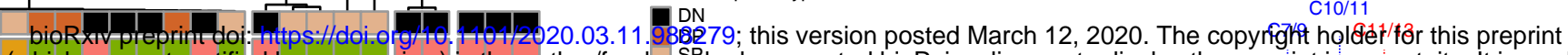
(which was not certified by peer review) is the author/funder, SWho has granted bioRxiv a license to display the preprint in perpetuity. It is made

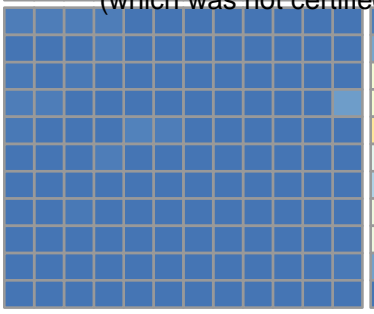

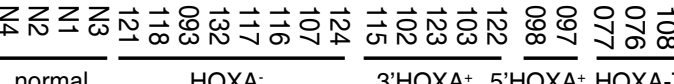

$\begin{array}{ll}\text { HOXA2 } & \text { ETP } \\ \text { HOXA3 } & \text { non-ETP } \\ \text { HOXA4 } & \text { normal } \\ \text { HOXA5 } & \text { gene expression } \\ \text { HOXA6 } & \begin{array}{l}\left(\log _{2} \text { FPKM }\right) \\ \text { HOXA7 }\end{array} \\ \text { HOXA9 } & 8 \\ \text { HOXA10 } & 6 \\ \text { HOXA11 } & \\ \text { HOXA13 } & 4\end{array}$

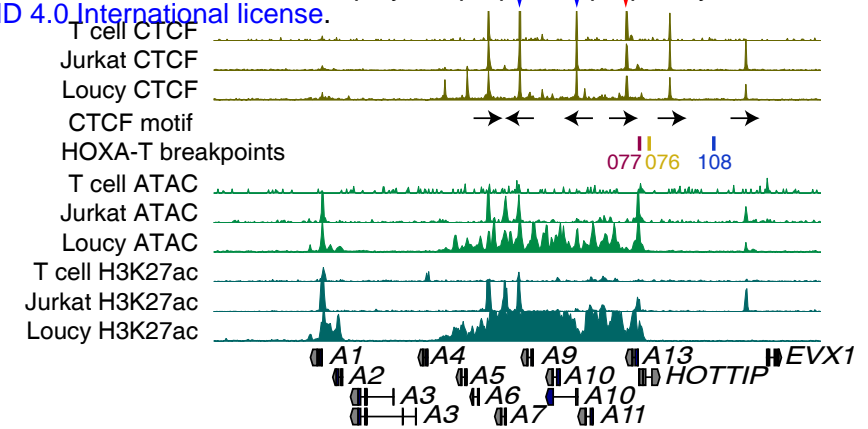

C

chr7

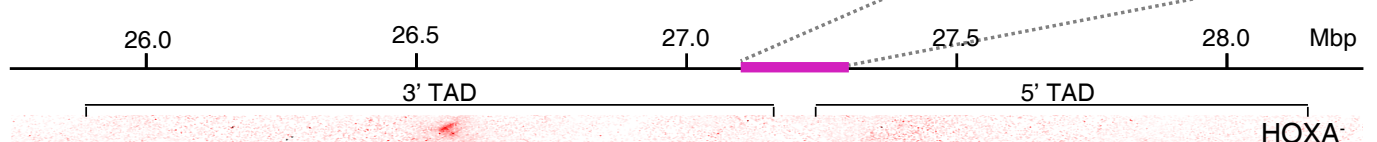

normalized interaction frenquency 10

differential interaction frenquency $\begin{array}{lll}-5 & 0 & 5\end{array}$
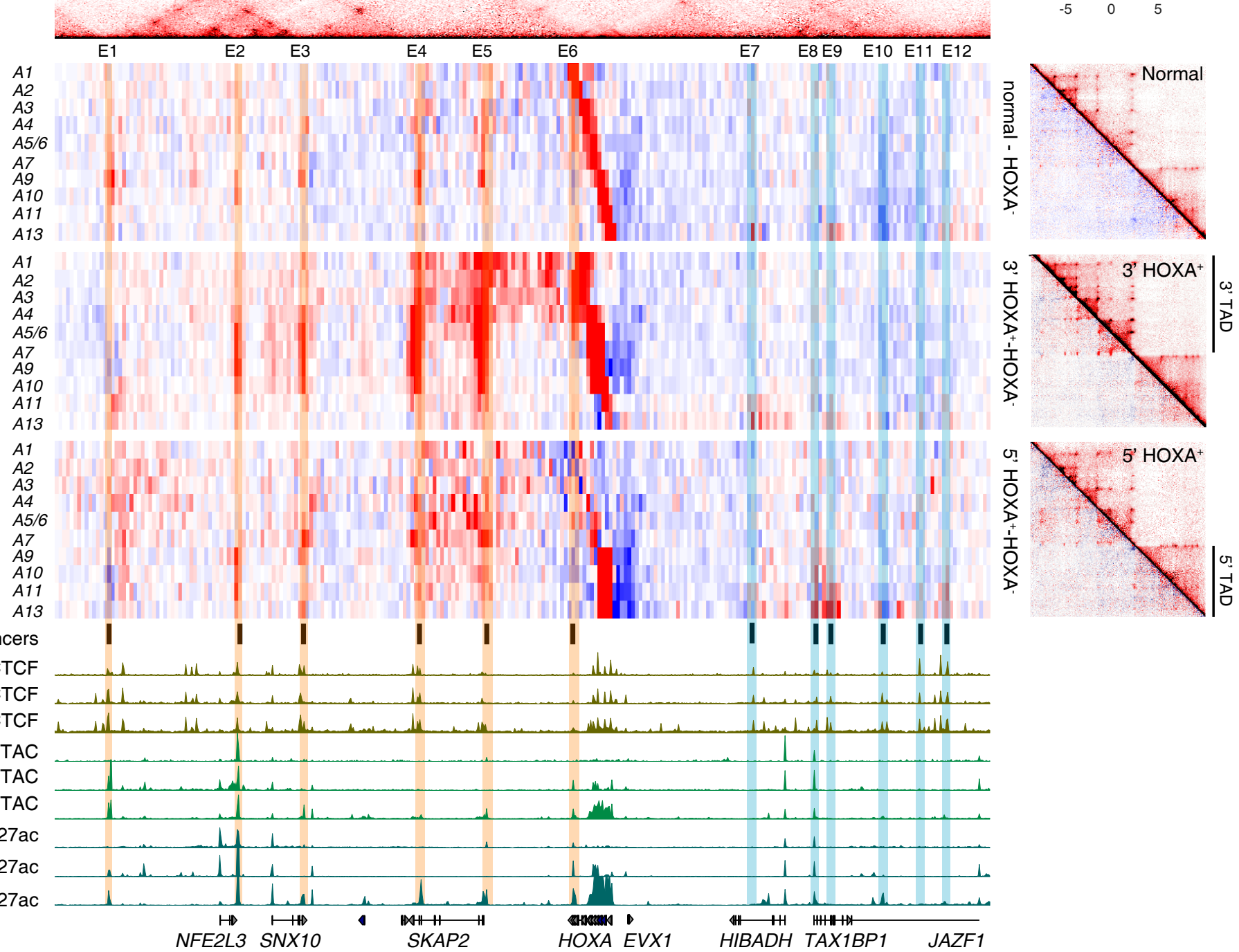

enhancers

T cell CTCF Jurkat CTCF

Loucy CTCF

$T$ cell ATAC Jurkat ATAC Loucy ATAC

T cell H3K27ac Jurkat H3K27ac Loucy H3K27ac NFE2L3 SNX10 SKAP2

5' $\mathrm{HOXA}^{+}$

3' TAD

5' TAD

E2 E3 E4 E5 E6 A1-7 A9A10 A11 A13 E8 E9 E11E12

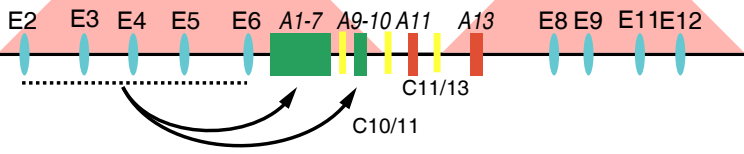

enhanced interactions

KMT2A-MLLT1 102

PICALM-MLLT10 103

DDX3X-MLLT10 123

SET-NUP214 122 enhancer CTCF silent gene active gene

enhanced interactions

PSIP1- NUP98 098 
bioRxiv preprint doi: https://doi.org/10.1101/2020.03.11.988279; this version posted March 12, 2020. The copyright holder for this preprint (which was not certified by peer review) is the author/funder, who has granted bioRxiv a license to display the preprint in perpetuity. It is made Fig. 4 Chromatin interaction profile and expression patterns of the HOXA cluster

in T-ALLs. (a) T-ALL subtypes with different HOXA gene expression patterns. HOXAtranslocation (HOXA-T) negative cases are grouped by unsupervised hierarchical clustering based on the HOXA gene expressions. (b) ChIP-seq tracks of CTCF and H3K27ac and ATAC-seq tracks in T cell, HOXA- Jurkat and HOXA ${ }^{+}$Loucy T-ALL cells in the genomic region corresponding to the pink bar of Fig. 4c. CTCF motif orientations, 5'TAD and 3'TAD boundaries and HOXA-T breakpoints are also included. (c) Top: a Hi-C heatmap shows the average interaction intensity of $\mathrm{HOXA}^{-}$cases in chr7: 25,750,000-28,250,000 (hg19), which includes HOXA gene cluster and its 3' and 5' TADs. Middle left: from top to bottom, Hi-C heatmaps show differential interaction intensities between normal T cell vs. $\mathrm{HOXA}^{-}, 3^{\prime} \mathrm{HOXA}^{+}$vs. $\mathrm{HOXA}^{-}$and $5^{\prime} \mathrm{HOXA}^{+}$vs. $\mathrm{HOXA}^{-}$cases using visual $4 \mathrm{C}$ plot. Each line represents interactome of a HOXA gene shown on the left. HOXA5 and HOXA6 share one line as they located in the same bin. The main enhancers are highlighted with orange in the 3' TAD and blue in the 5' TAD. Middle right: Top right of each heatmap represents the averaged interaction intensities of normal T cell, 3' $\mathrm{HOXA}^{+}$cases and 5' $\mathrm{HOXA}^{+}$cases, respectively; bottom left of each heatmap represents the differential interaction intensities between each group and HOXA cases. Bottom: ChIP-seq tracks for CTCF and H3K27ac and ATAC-seq tracks in T cell, HOXA- Jurkat and HOXA ${ }^{+}$Loucy T-ALL cells. (d) Schematic illustrations of the associations among 3D genomic interactions, HOXA cluster expressions and associated fusion events in 3' HOXA cases (left) and 5' HOXA cases (right). 


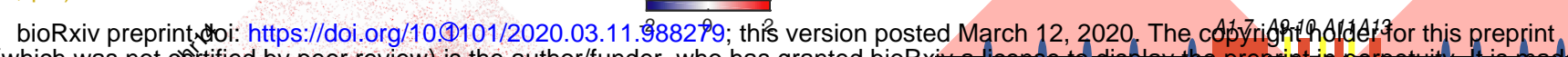

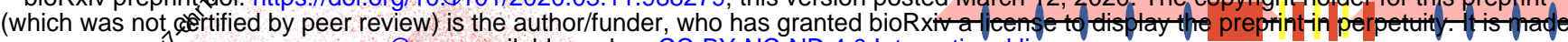

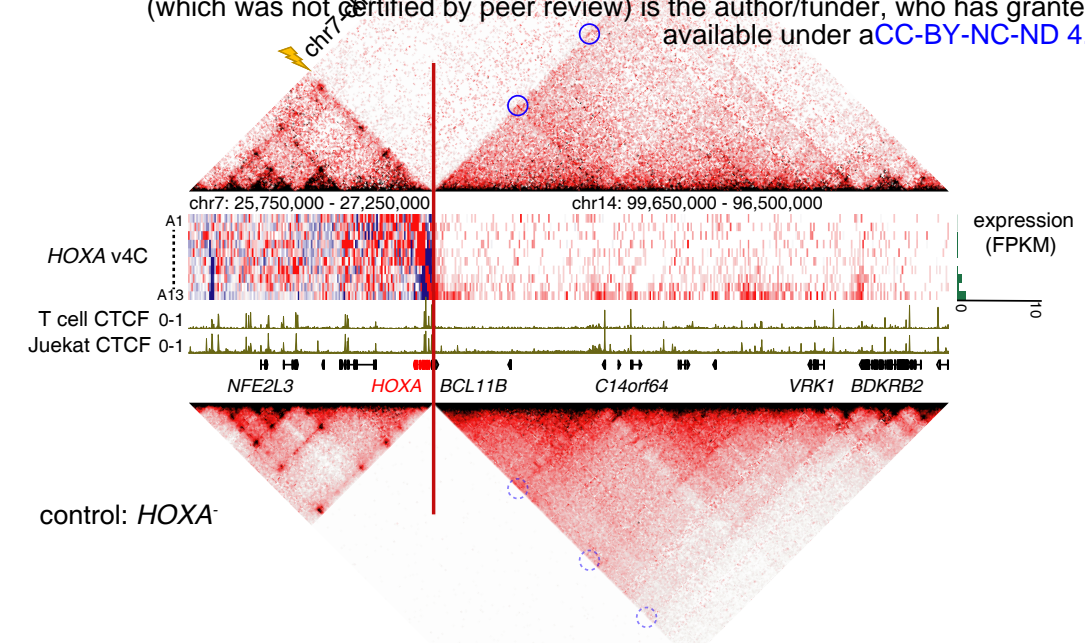

b

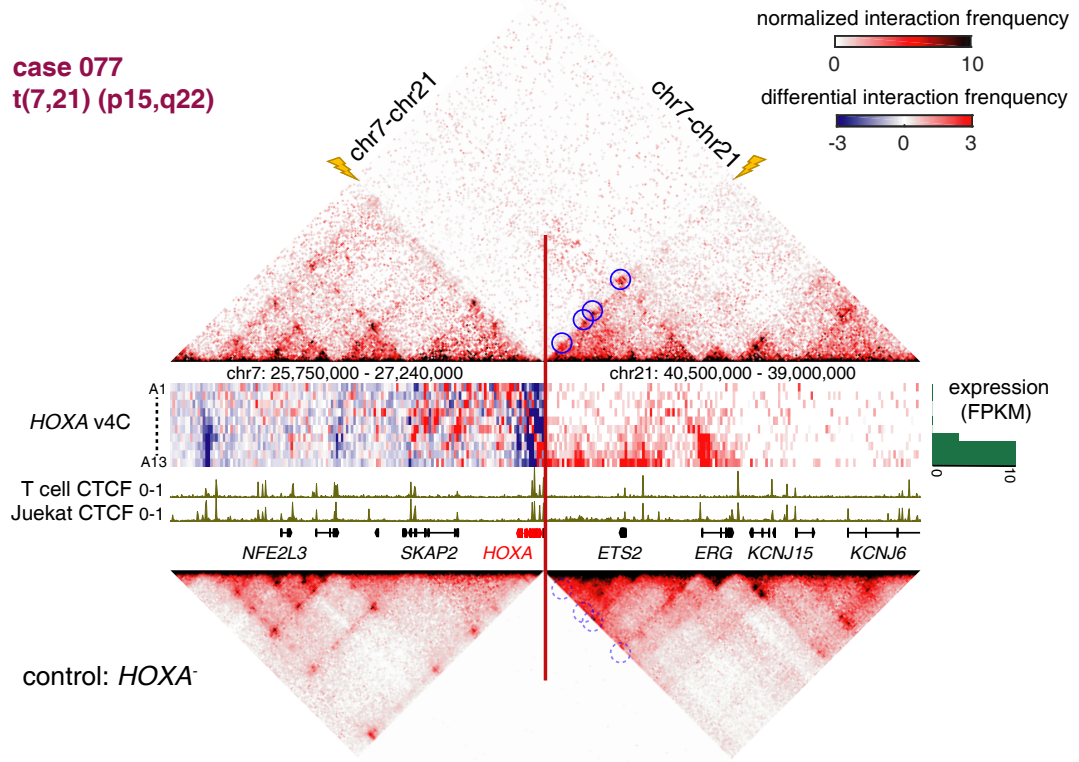

C

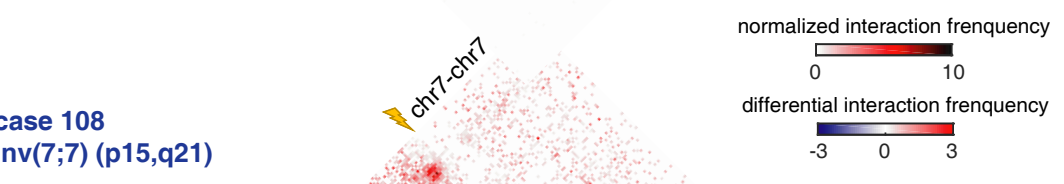

HOXA v4C

T cell CTCF A13
$0-1$ a 19 Ju

inv $(7 ; 7)(p 15, q 21)$
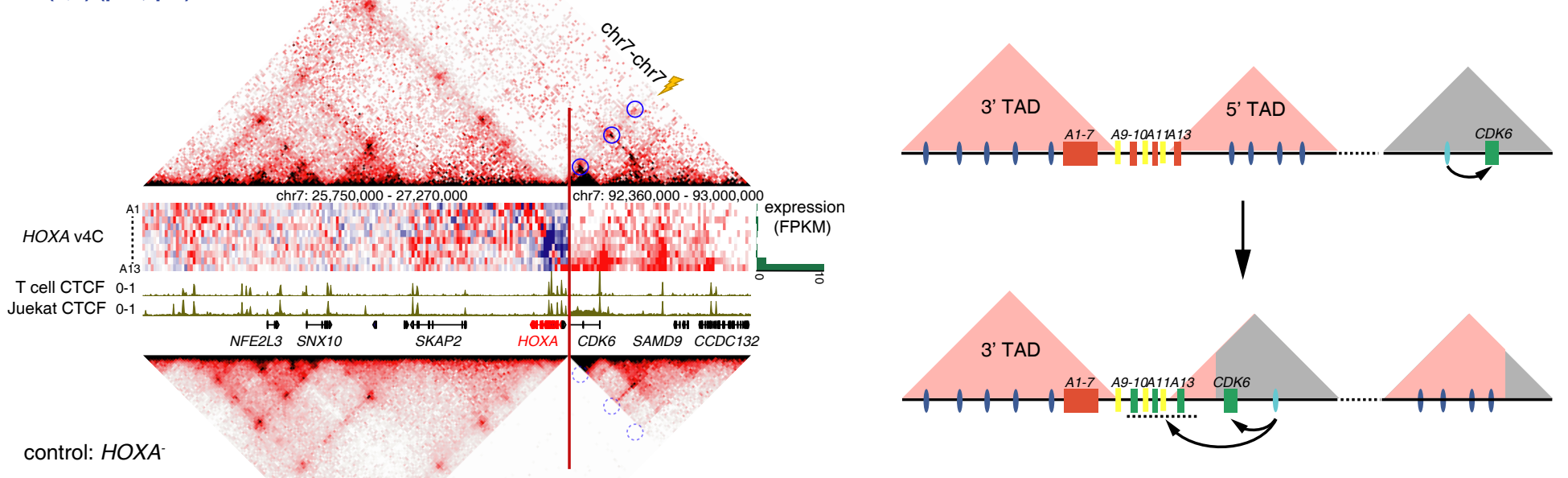
bioRxiv preprint doi: https://doi.org/10.1101/2020.03.11.988279; this version posted March 12, 2020. The copyright holder for this preprint (which was not certified by peer review) is the author/funder, who has granted bioRxiv a license to display the preprint in perpetuity. It is made

\section{Fig. 5. Translocation-mediated enhancer hijacking and ectopic HOXA gene}

expressions in T-ALLs. (a-c) Individual Hi-C heatmaps for three HOXA-T cases (top) and averaged Hi-C heatmaps of $\mathrm{HOXA}^{-}$control cases (bottom). Middle panels are differential visual 4C plots between HOXA-T cases and HOXA- using HOXA1-A13 genes (from top to bottom) as the viewpoint. The green bar graphs on the right show the expression level of each HOXA gene. Breakpoints are marked by the red lines and yellow lightning bolts. Translocation-mediated loops are highlighted by blue circles, corresponding locations in control samples are marked by dotted circles. (d) Schematic illustrations of the consequences of HOXA-related translocations (upper) and inversion (lower). 
a

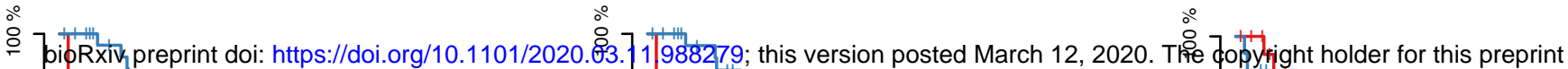

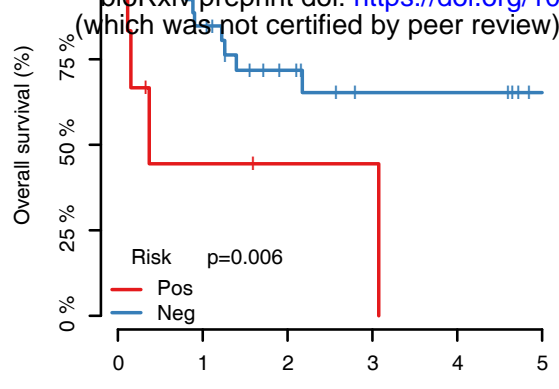
availabse ginder aCC-BY-NC-ND 4.0 International license.

Number at risk

High: 6

Time (years)

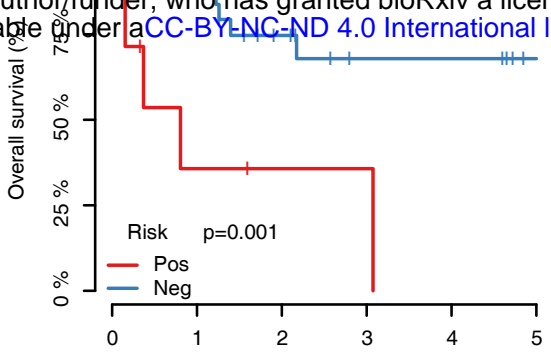

Number at risk

High: 7

Time (years)

$\begin{array}{cc} & \text { Time (ye } \\ 2 & 1 \\ 22 & 13\end{array}$

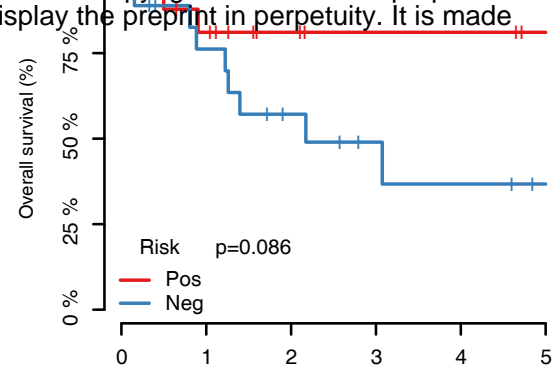

Number at risk

High: 20

Time (years)

b

total DN ETP

C
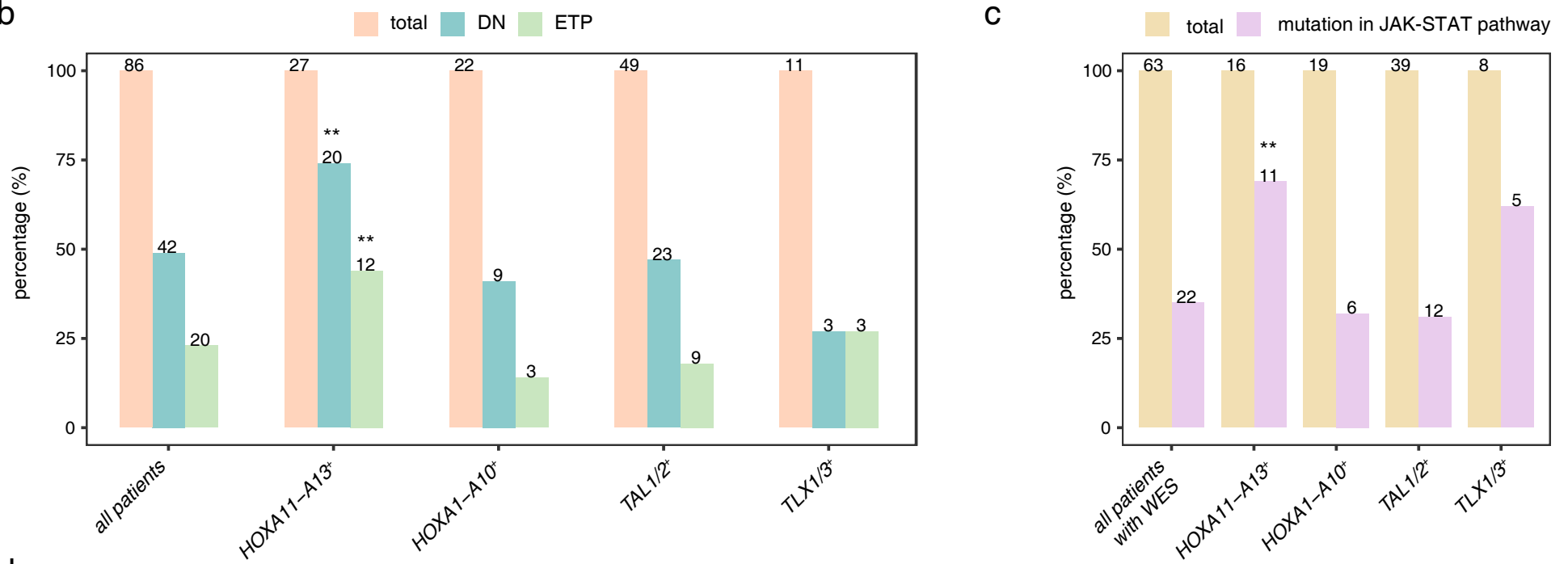

$d$

\begin{tabular}{|c|c|c|c|c|c|c|c|c|c|}
\hline & \multicolumn{3}{|c|}{ Univariate } & \multicolumn{3}{|c|}{ Multivariate (HOXA13) } & \multicolumn{3}{|c|}{ Multivariate (HOXA11-13) } \\
\hline & $\mathrm{HR}$ & $95 \% \mathrm{Cl}$ & $P$ value & $\mathrm{HR}$ & $95 \% \mathrm{Cl}$ & $\mathrm{P}$ value & $\mathrm{HR}$ & $95 \% \mathrm{Cl}$ & $\mathrm{P}$ value \\
\hline $\begin{array}{l}\text { HOXA13 expression } \\
\qquad \begin{array}{c}(\mathrm{FPKM}) \\
\geq 1 \text { vs. }<1\end{array}\end{array}$ & 4.675 & $1.39-15.69$ & 0.013 & 38.25 & $1.37-1071$ & 0.032 & & & \\
\hline $\begin{array}{l}\text { HOXA11-13 } \\
\text { expression } \\
\text { (FPKM) } \\
\geq 1 \text { vs. }<1\end{array}$ & 5.872 & $1.84-18.74$ & 0.003 & & & & 8.84 & $0.82-95.24$ & 0.072 \\
\hline $\begin{array}{c}\text { Gender } \\
\text { Males vs. Female }\end{array}$ & 1.13 & $0.42-3.01$ & 0.806 & 0.959 & $0.05-17.78$ & 0.978 & 0.89 & $0.066-12.015$ & 0.93 \\
\hline $\begin{array}{c}\text { Age } \\
\text { Pediatric vs. Adult }\end{array}$ & 0.21 & $0.07-0.64$ & 0.006 & 0.154 & $0.006-3.684$ & 0.248 & 0.34 & $0.014-8.444$ & 0.512 \\
\hline $\begin{array}{c}\text { WBC } \\
\text { Low vs. High }\end{array}$ & 1.46 & $0.62-3.43$ & 0.381 & 73.52 & $1.59-3390$ & 0.028 & 31.95 & $1.5-680.5$ & 0.026 \\
\hline Hemoglobin & 1 & $0.99-1.02$ & 0.571 & 1.02 & $0.94-1.11$ & 0.574 & 1.008 & $0.94-1.077$ & 0.813 \\
\hline Platelet & 1 & $0.99-1$ & 0.952 & 0.986 & $0.968-1.004$ & 0.127 & 0.987 & $0.971-1.004$ & 0.139 \\
\hline $\begin{array}{c}\text { Hepatosplenomegaly } \\
\text { Yes vs. No }\end{array}$ & 1.47 & $0.34-6.33$ & 0.605 & 0.24 & $0.004-15.882$ & 0.504 & 1.67 & $0.022-128.414$ & 0.817 \\
\hline Blasts in BM & 1.01 & $0.98-1.03$ & 0.621 & 1.107 & $0.906-1.352$ & 0.319 & 1.08 & $0.9-1.294$ & 0.407 \\
\hline
\end{tabular}


bioRxiv preprint doi: https://doi.org/10.1101/2020.03.11.988279; this version posted March 12, 2020. The copyright holder for this preprint (which was not certified by peer review) is the author/funder, who has granted bioRxiv a license to display the preprint in perpetuity. It is made Fig. 6 Ectopic HOXA11-A13 expressions are correlated with poor outcomes in pediatric and young adult T-ALLs. (a) Kaplan-Meier overall survival curves for patients with (red) and without (blue) ectopic HOXA13 expression (left), HOXA11-A13 expressions (middle) and TAL1/TAL2 expressions (right) in pediatric and young adult patients. (b) The associations between ectopic HOXA11-A13 expression and DN and ETP phenotypes. $P$-values are calculated by Fisher exact test $(\star *: p<0.01)$. (c) The proportion of cases with JAK-STAT pathway mutations in each T-ALL subgroup, $P$ values are calculated by Fisher exact test $(* *$ p $p<0.01)$. (d) Univariable and multivariable analysis of overall survival. $\mathrm{HR}$, hazard ratio; $\mathrm{Cl}$, confidence interval. 